\title{
Duality, statistical mechanics, and random matrices
}

\author{
Thomas Spencer
}

\begin{abstract}
This article will present an informal review of some results and conjectures about the spectral theory of large random matrices and related spin systems in statistical mechanics. A class of lattice spin models provides a dual representation for spectral problems in random matrix theory. Ordered and disordered phases of the spins correspond to different spectral types and quantum time evolutions. In three dimensions, we describe a phase transition for a supersymmetric statistical mechanics system inspired by random matrix theory. This transition has a classical interpretation in terms of a history dependent walk on the lattice. In the ordered phase the walk is diffusive while in the disordered phase it is localized near its starting point.
\end{abstract}

\section{Contents}

1. Overview 229

2. SUSY statistical mechanics 242

3. A phase transition for the $H^{2 \mid 2}$ model $\quad 251$

4. Concluding Remarks 257

$\begin{array}{ll}\text { References } & 258\end{array}$

\section{Overview}

In this section we shall give a brief overview of conjectures and theorems about random matrices and related models of interacting lattice spins. We begin by defining various classes of large random matrices including Wigner matrices, random band matrices and the Schrödinger equation with a random potential. Spectral properties of these matrices such as density of states, eigenvalue spacing, time evolution and notions of localization and quantum diffusion are described in $\S 1.1, \S 1.2$ and $\S 1.4$. Some simple examples of duality appear in $\S 1.3$. In this case the dual statistical mechanics consists of a single "spin". Standard saddle point analysis yields precise asymptotic information. An introduction to supersymmetric spin models and their relation to the spectral theory of random matrices is explained in $\S 2$.

In $\S 1.6$ we review the ordered and disorded phases for some classical spin models of statistical mechanics. Symmetry is conjectured to describe some universal features of the ordered spin phase. In $\S 1.5$ and $\S 3$ we discuss a three dimensional 
supersymmetric spin model which has a phase transition. This supersymmetric model is equivalent to a history dependent walk called the vertex reinforced jump process. In the ordered phase the walk is diffusive while in the disordered phase it is localized near its starting point. A similar transition is believed to occur for the random Schrödinger equation.

1.1. Wigner Matrices. There has been a great deal of interest and activity in the spectral theory of random matrices arising from various branches of mathematics and physics. These areas include probability, statistics, number theory, combinatorics, communication theory, quantum chaos and the spectral theory of Schrödinger operators with a random potential. See [Bre] for a review of some of these applications.

The study of large random matrices in physics originated with the work of Eugene Wigner in the 1950's who used them to predict the energy level statistics of a large nucleus. He argued that because of the complex interactions in the nucleus there should be a random matrix model with appropriate symmetries, whose eigenvalues would describe the energy level spacing statistics. He introduced a class of $N \times N$ random Hermitian $H=H^{*}$ and real symmetric matrices, $H=H^{t}$, called Wigner matrices. These matrices have a mean field structure: their matrix elements are independent and have equal variance subject to symmetry constraints. In particular for Hermitian matrices

$$
\left\langle H_{i j}\right\rangle=0 \quad \text { and } \quad\left\langle H_{i j} H_{i^{\prime} j^{\prime}}\right\rangle=\left\langle H_{i j} \bar{H}_{j^{\prime} i^{\prime}}\right\rangle=\frac{1}{N} \delta_{i j^{\prime}} \delta_{j i^{\prime}} .
$$

In the special case where the matrix elements have a Gaussian distribution these conditions uniquely specify the probability distribution and the ensemble is referred to as the Gaussian Unitary Ensemble (GUE). Its probability distribution is proportional to $e^{-N \operatorname{Tr} H^{2} / 2}$. The Gaussian Orthogonal ensemble (GOE) has the same weight except that we integrate over real symmetric matrices.

For a large class of matrices satisfying (1.1), most of the eigenvalues lie in the interval $[-2,2]$ as $N \rightarrow \infty$. Wigner [Wig1] used the method of moments to show that as $N \rightarrow \infty$ the fraction of eigenvalues of $\mathrm{H}$ less than $\mathrm{E}$ is given by $\int_{-2}^{E} \rho\left(E^{\prime}\right) d E^{\prime}$ where the density of states (eigenvalues) $\rho(E)$ satisfies the semicircle law:

$$
\rho(E)=\frac{1}{2 \pi} \sqrt{4-E^{2}}, \quad|E|<2 .
$$

The density of states can be defined for a broad class of random matrices. It is not universal because it depends on the details of the random matrix distribution.

Universality: Wigner and Dyson conjectured that the statistics of suitably scaled eigenvalue spacings such as $\left(E_{k+1}-E_{k}\right) / \delta(E)$ is universal for many large complex quantum systems. The scaling factor $\delta(E)=(\rho(E) N)^{-1}$ is the average eigenvalue spacing about E. Universality means that such spacing statistics should only depend on symmetry (Hermitian GUE or real Symmetric GOE or Symplectic GSE). These statistics are often referred to as Wigner-Dyson statistics and are observed in many physical systems. See [Dys] and [Wig2] for Wigner's historical perspective. Universality implies that the simpler mean field Gaussian models should accurately predict local spacing laws for a broad class of quantum ensembles. For large N GUE matrices, the local eigenvalue correlation $\rho_{2}$ is given by

$$
\frac{1}{\rho^{2}(E)} \rho_{2}(E, E+\xi \delta(E))=1-\sin ^{2}(\pi \xi) /(\pi \xi)^{2}, \quad|E|<2
$$


For small $\xi$ we see that there is quadratic repulsion between nearby eigenvalues. The spacing distribution is more complicated and is expressed in terms of a Fredholm determinant.

Saddle manifold approximation. One of the goals of this article is to explain how Wigner-Dyson universality has a natural interpretation in terms of interacting spins or fields. We shall see that these spin systems are associated to a symmetric space related to the symmetry of the random matrix. Many lattice spin systems have a phase transition as temperature is varied. At high temperature, the spins are disordered whereas at low temperature they tend to be aligned or ordered in 3 or more dimensions. This is much like an iron magnet which when heated loses its ordered magnetic properties. In $\S 1.6$ the ordered (low temperature) phases of a wide class of spin models on $\mathbb{Z}^{d}, d \geq 3$ are conjectured to be described by the saddle manifold or 0-mode approximation. This is a mean field model determined by symmetry. To investigate a large random matrix near energy E, a dual spin system is studied at a temperature $\mathrm{T}(\mathrm{E})$. In the framework of supersymmetric statistical mechanics W-D statistics arise from the contribution of finite dimensional saddle manifold of spins in an ordered state, [Ef1, Ef2].

Let us remark that universality of mean field theory and the saddle manifold discussed in this article is different from the more subtle notion of universality in critical phenomena. Critical universality occurs at temperatures very near the transition temperature which marks the borderline between order and disorder.

Number Theory: Wigner-Dyson statistics seem to extend far beyond spectral theory of random matrices. The high zeros of the Riemann zeta function appear to satisfy Wigner-Dyson statistics for GUE after suitable scaling. This striking conjecture is due to H. Montgomery and F. Dyson followed by numerical work of A. Odlyzko. See [RS, KS] for further developments relating number theory to random matrices.

Mathematical Results on universality: Recent work by Erdős et al. [Er2] and by Tao and $\mathrm{Vu}[\mathrm{TV}]$ established universality of spacing statistics for a wide class of Wigner matrices. This may be thought of as a noncommutatitve central limit theorem. Note that Wigner-Dyson describes the law of highly correlated random variables namely the eigenvalues of a random matrix. A more detailed review of these developments as well as recent results on sparse and band matrices is covered in the lectures of L. Erdös [Er5] and in [Er4].

There is a related family of matrices called invariant ensembles. In this case the matrix elements are given by a probability density of the form $e^{-N \operatorname{tr} V(H)}$ where $V(x) \geq 0$ is a function with suitable growth for large $|x|$. Unless $V(x)=x^{2}$, the matrix elements are no longer independent and the density of states is not given by the semi-circle law. Universality has also been established for invariant ensembles by Deift et al [Dei] and by Pastur and Shcherbina [PS1, PS2]. This means that the eigenvalue spacing distribution does not depend on $\mathrm{V}$ but it will depend on whether the matrices are complex Hermitian or real symmetric. This has lead to progress in areas such as Riemann-Hilbert theory, orthogonal polynomials, integrable systems, matrix-large deviation theory. See also recent work of Bourgade, Erdős, and Yau [BEY] which generalizes this work to a more general class of statistical mechanics models with a logarithmic potential.

1.2. The Anderson model and Random Band matrices. A few years after Wigner's work on energy levels of a large nucleus, Philip Anderson [And] 
introduced another class of random matrices to study a quantum particle on a d dimensional crystal which is scattered by random defects or impurities. The Anderson model is given by a discrete space Schrödinger operator on $\mathbb{Z}^{d}$ with a random potential V:

$$
H=-\Delta+\lambda V \quad(H w)(j)=\sum_{j^{\prime}:\left|j^{\prime}-j\right|=1}\left(w(j)-w\left(j^{\prime}\right)\right)+\lambda V(j) w(j) \quad j \in \mathbb{Z}^{d} .
$$

The $V_{j}$ are usually assumed to be independent, identically distributed random variables with mean 0 and unit variance. The coupling $\lambda$ measures the strength of the disorder. For $\lambda \gg 1$ Anderson predicted localization with probability one for all energies E. An eigenstate $\phi$ is said to be localized if it decays exponentially fast about some lattice point $\mathrm{b}$,

$$
|\phi(j)| \approx C e^{-|j-b| / \ell(E)}
$$

where $\ell(E)$ is called the localization length. Mathematically, localization at all energies means that with probablity one, the spectrum of $\mathrm{H}$ is dense, pure point spectrum, and eigenstates decay exponentially fast. Note that if we drop the discrete Laplacian in (1.4) each eigenstate is concentrated at a single lattice vertex. In physics, localization corresponds to an insulating phase - no conduction. The Anderson model is also be studied on $\mathbb{R}^{d}$ and its low energy spectral behavior will be much the same as on the lattice.

Goldsheid, Molchanov and Pastur [GMP] proved localization for all energies in one dimension for any $\lambda>0$. Thus even small amounts of disorder changes the character of the eigenstates in 1D from plane wave to localized. For $d \geq 2$ localization was proved using the estimates of [FS2] for strong disorder $\lambda \gg 1$ or for small $\lambda>0$ and $E \leq-C \lambda^{2}$. See [AM] for an elegant proof using fractional moments and $[\mathrm{Sp} 1, \mathrm{Sto}]$ for mathematical reviews of Anderson localization.

The Anderson transition is expected to occur in three dimensions for small disorder (eg. $|\lambda| \leq 1)$. There is conjectured to be an interval of absolutely continuous spectrum $\left(E_{m}, E_{m}^{\prime}\right)$ corresponding to energies where conduction or quantum diffusion occurs. For E outside the interval, $\ell(E)<\infty$, and $\ell(E)=\infty$ inside the interval. The energies $E_{m}$ and $E_{m}^{\prime}$ are well defined with probability one and depend on $\lambda$. They are called mobility edges. Although the existence of such an interval is not questioned in theoretical physics, a mathematical proof of its existence has not been established and is considered to be a major open problem. It will be rephrased more precisely in terms of Green's functions (1.16) below.

In statistical mechanics the role of $\mathrm{E}$ is replaced by temperature $\mathrm{T}(\mathrm{E})$. Localization corresponds to a high temperature $\mathrm{T}(\mathrm{E})$ phase where spins are disordered whereas quantum diffusion corresponds to an ordered phase, low temperature. In two dimensions, Abrahams, Anderson, Liciardello and Ramakrishnan [Abr] predicted that for any $\lambda>0$ all states are localized with a localization length $\ell \approx e^{c \lambda^{-2}}$. See $[\mathrm{EM}]$ for a recent review of the predicted behavior of eigenfunctions with $E \approx E_{m}$ using supersymmetric statistical mechanics.

Random Band Matrices: This article will focus on spectral properties of large Hermitian random band matrices, RBM. These matrices, $H_{i j}=\bar{H}_{j i}$ are indexed by $i, j$ in $\mathbb{Z}^{d}$ and concentrated in a band of width $\mathrm{W}$ about the diagonal, $|i-j| \leq$ $W$. They interpolate between the mean field Wigner type matrices (when $W \approx$ $N$ ) and the Anderson model. The Anderson model and the random band model with fixed $\mathrm{W}$ are expected to have very similar behavior. There is an approximate 
correspondence $\lambda \approx 1 / W$. Note however that the Anderson model is real symmetric (time reversal invariant) whereas RBM we study are complex Hermitian and thus they belong to distinct Wigner-Dyson classes.

Definition of $R B M$ : We shall assume that $H_{i j}$ are independent random variables of zero mean subject to the Hermitian constraint. In addition we assume that $H_{j k}$ are Gaussian and

$$
\left\langle H_{i j}\right\rangle=0 \quad \text { and } \quad\left\langle H_{i j} H_{i^{\prime} j^{\prime}}\right\rangle=J_{i j} \delta_{i j^{\prime}} \delta_{j i^{\prime}} \quad i, j \in \mathbb{Z}^{d} \cap[1, N]^{d} \equiv \Lambda_{N}
$$

with $J_{i j} \approx 0$ when $|i-j| \geq W$ and normalized so that $\sum_{j} J_{i j}=1$. W is called the width of the band. For fixed $\mathrm{W}$ and large N, RBM reflects the geometry of the lattice. The special case where $W=N$ and $J_{i j}=1 / N$, defines the Gaussian unitary ensemble (GUE).

Topics of particular interest are the density of states, the statistical properties of eigenvalues and eigenvectors of $\mathrm{H}$ when $\mathrm{H}$ is restricted to a large box of side $\mathrm{N}$. As in the Anderson model a closely related topic is a description of the long time evolution of the Schrödinger equation on $\mathbb{Z}^{d}$

$$
i \partial \phi(t, j) / \partial t=H \phi(t, j)
$$

given an initial state $\phi(0, j)$. The time evolution $(\S 1.4)$ will reflect localized and conducting states depending upon whether the initial state spreads.

Although there is a wealth of information for mean field models, the study of RBM is much less developed. For one dimensional RBM, Schenker [Sch] proved that the localization length $\ell(E) \leq W^{8}$ for all E. Using SUSY statistical mechanics, Fyodorov and Mirlin $[\mathrm{FM}]$ predicted that the length is $\approx W^{2}$. Sodin [Sod] proved that the low eigenvalues of a random band matrix form an Airy process which coincides with that of GUE or GOE provided $W \gg N^{5 / 6}$. His result is sharp and in particular it implies that the lowest eigenvalue fluctuates following the TracyWidom distribution. Recent work of Erdös, Knowles, Yau and Yin [Er3] proves results about eigenstates and diffusive time evolution for $W^{5 / 4} \gg N$. Wigner-Dyson statistics for local eigenvalue correlations are expected to occur for $E \in(-2,2)$ provided that $W^{2} \gg N \gg 1$, [FM]. In three dimensions Wigner-Dyson statistics should hold for $W \gg 1$ fixed as $\mathrm{N}$ gets large. See [Er4, Sp2] for recent mathematical reviews of sparse matrices and RBM.

1.3. Duality and Green's functions. The statistical mechanics approach to the spectral theory of random matrices described in these lectures was pioneered by physicists F. Wegner [Weg], L. Schäffer [SW] and K. Efetov [Efe1] in the early 1980's. It extracts spectral information of RBM in terms of certain supersymmetric (SUSY) statistical lattice spin models. The word supersymmetry refers to the fact that the spins have both Grassmann (anticommuting) and real components which appear in a symmetric fashion. $\S 2$ gives a brief introduction to Grassmann variables and supersymmetric models. In theoretical physics there are a number of reviews of SUSY and random matrices, [Efe2, Ef4, Fyo, Mir, VWZ, Zir1]. This mathematical review has considerable overlap with [Sp3].

We will see that there is a precise duality between SUSY statistical mechanics and RBM. The mathematical challenge is to analyze the corresponding SUSY statistical mechanics model and to prove that spins are aligned at long distances. There are similar mathematical problems for the quantum ferromagnet, superfluid 
and Bose-Einstein condensation.

We begin with a simple example of our notion of duality given by Stirling's formula:

$$
N !=\int_{0}^{\infty} e^{-t} t^{N} d t=N^{N+1} \int_{0}^{\infty} e^{-N[s-\ln s]} d s \approx N^{N} e^{-N} \sqrt{2 \pi N} .
$$

To obtain the right side we set $t=N s$, and note that the integrand is maximized for $s^{*}=1$. Fluctuations about the maximum of $-[s-\ln s]=-1-(s-1)^{2} / 2+\ldots$ are Gaussian as indicated by the second term of the expansion. The right side of (1.8) follows from the identity $\int e^{-N s^{2} / 2} d s=\sqrt{2 \pi / N}$. Higher order terms produce corrections of order $1 / \mathrm{N}$.

A more sophisticated version of duality is the asymptotic formula for counting the number of partitions of a large integer $\mathrm{N}$ given by Hardy and Ramanujan [HR]:

$$
P(N)=\frac{1}{2 \pi i} \oint \frac{1}{\prod_{m}\left(1-z^{m}\right)} z^{-N-1} d z \approx \frac{1}{4 \sqrt{3} N} e^{\pi \sqrt{2 N / 3}} .
$$

In this case, the complex variable $z$ is on a contour about 0 lying inside $|z|=1$. The saddle points depend upon $\mathrm{N}$ and are close to the roots of unity. A detailed analysis of this integral uses the theory of modular forms. See [Apo] for an exposition of Rademacher's work, [Rad].

To illustrate a simple form of duality for random matrices, let $\mathrm{H}$ be an $\mathrm{N}$ by $\mathrm{N}$ Hermitian matrix with the GUE ( Gaussian Unitary Ensemble) distribution proportional to $e^{-N T r H^{2} / 2}$. The Gaussian average of the determinant of the resolvent, $\left(E_{\varepsilon}-H\right)^{-1}$ can be expressed as

$$
\left\langle\operatorname{det}\left(E_{\varepsilon}-H\right)^{-1}\right\rangle=\frac{(-i N)^{N}}{(N-1) !} \int_{0}^{\infty} e^{-N\left(s^{2} / 2+i E_{\varepsilon} s\right)} s^{N} d s / s, \quad E_{\varepsilon} \equiv E-i \varepsilon, \quad \varepsilon>0 .
$$

For large $\mathrm{N}$ and $|E|<2$ this integral is dominated by the contributions near its saddle point $s^{*}=-i E / 2+\sqrt{1-(E / 2)^{2}}$. To analyze the right side we deform the contour of integration so that it passes through $s^{*}$ and the integrand achieves it maximum modulus there. Then expand to second order about $s^{*}$ as in Stirling's formula.

Key Identities: The derivation of (1.10) will be given in $\S(2.1)$. It relies on the unitary invariance of $e^{-N T r H^{2} / 2}$ and the identity

$$
\pi^{N} \operatorname{det} M^{-1}=\int e^{-z^{*} \cdot M z} \prod_{i=1}^{N} d x_{i} d y_{i} .
$$

Above we have set $z_{j}=x_{j}+i y_{j}, 1 \leq j \leq N$ and assumed that $\mathrm{M}$ is an $\mathrm{N}$ by $\mathrm{N}$ matrix with $\operatorname{Re} M>0$ so that the integral is well defined. There is a similar expression for the determinant of $\mathrm{M}$ using Grassmann variables $\bar{\psi}, \psi$

$$
\operatorname{det} M=\int e^{-\bar{\psi} \cdot M \psi} \prod_{i=1}^{N} d \bar{\psi}_{i} d \psi_{i} .
$$

See $\S(2.1)$ for properties of the anticommuting variables $\bar{\psi}_{i}, \psi_{i}$. These formulas will play a key role in the SUSY formulation of random matrices by setting $M=$ $i\left(E_{\varepsilon}-H\right)$. If $\mathrm{H}$ is Gaussian we can calculate the expectation of (1.11) and (1.12) explicitly in terms of $\bar{z}, z$ and $\bar{\psi}, \psi$. 
Statistical Mechanics: Duality for more general Gaussian N by N random matrices, such as band matrices, involves integrals of many spin variables rather than the single spin $s$ above. This is the domain of statistical mechanics which naturally suggests the use of nonperturbative techniques such as steepest descent, collective coordinates and renormalization to analyze spectral properties of random matrices. We shall see that unexpected symmetries also emerge in statistical mechanics formulation of RBM and these will help explain Wigner-Dyson universality of local eigenvalue statistics.

Green's Functions: In order to investigate the spectral properties of a random matrix $H$ one studies averages of the resolvent or Green's function

$$
G\left(E_{\varepsilon} ; j, k\right)=\left(E_{\varepsilon}-H\right)^{-1}(j, k) \quad E_{\varepsilon}=E-i \varepsilon, \quad \varepsilon>0
$$

where $\mathrm{E}$ is real and $j, k \in Z^{d} \cap[-N, N]^{d}$ label the matrix elements. Note $\operatorname{Im}[(E-$ $x)-i \varepsilon]^{-1} \approx \pi \delta_{\varepsilon}(E-x)$, where $\delta_{\varepsilon}$ is a regularized Dirac delta function. Thus the imaginary part of the Green's function gives spectral information about $\mathrm{H}$ in an $\varepsilon$ neighborhood of E. The ultimate goal is to study $G$ for $\varepsilon \approx N^{-d}$ which is the typical separation of eigenvalues. In order to understand evolution of the quantum dynamics up to time $\mathrm{T}$, we need good estimates on $\mathrm{G}$ for $\varepsilon^{-1} \approx T$. The identity

$$
\pi \rho_{\varepsilon}(E)=\operatorname{Im}\left\langle G\left(E_{\varepsilon} ; 0,0\right)\right\rangle=\varepsilon \sum_{j}\left\langle\left|G\left(E_{\varepsilon} ; 0, j\right)\right|^{2}\right\rangle
$$

is easily proved by applying the resolvent identity to $G-\bar{G}$. It reflects conservation of probability or unitarity and is some times referred to as a Ward identity.

Note that by Cramer's rule, Green's functions may be expressed as a ratio of determinants. This is why identities (1.11) and (1.12) are useful. We shall see that the average Green's function may be identified with the correlation of a spin $S_{j}$ and $S_{k}$, at temperature $\mathrm{T}(\mathrm{E})$. The Gibbs weight for the corresponding supersymmetric statistical mechanics is generally rather complicated and will later be described in special cases.

In $\S 2.1$ and $\S 2.2$ we shall explain how to use statistical mechanics to get estimates on the density of states $\rho(E)$ for GUE and random band matrices. The density of states can be expressed as

$$
\pi \rho(E)=\lim \varepsilon \downarrow 0 \operatorname{Im}\left\langle G\left(E_{\varepsilon} ; 0,0\right)\right\rangle .
$$

To obtain information about the eigenstates or time evolution we must analyze the average of the modulus squared of the Green's function which distinguishes localization and diffusion. This is considerably more difficult to analyze than (1.15). These are two basic conjectured scenarios which will depend on the dimension of the lattice, $\mathrm{W}$ and the energy $\mathrm{E}$ :

Extended States and quantum-diffusion in 3D:

$$
\left\langle\left|G\left(E_{\varepsilon} ; j, k\right)\right|^{2}\right\rangle \cong \frac{\rho(E)}{-D \Delta+\varepsilon}(j, k) \approx C(|j-k|+1)^{-1} .
$$

for $j, k \in \mathbb{Z}^{3}$ and $E \in\left(E_{m}, E_{m}^{\prime}\right)$. For $\mathrm{RBM}$ in $3 \mathrm{D}$ we expect $E_{m} \approx-2$ and $E_{m}^{\prime} \approx 2$ for large W. Note the right side is proportional to the Laplace transform of the heat kernel. The quantum particles of energy $\mathrm{E}$ are conducting states and thus correspond to the metallic phase. In a large finite box the eigenstates are uniformly spread out. GUE Wigner-Dyson statistics should govern the eigenvalue spacings near E. For the real symmetric Anderson model (1.4), these statistics 
should be given in terms of GOE matrices. In infinite volume, energies for which $\left\langle\left|G\left(E_{\varepsilon} ; j, j\right)\right|^{2}\right\rangle$ is bounded as $\varepsilon \downarrow 0$ belong to the absolutely continuous spectrum of $\mathrm{H}$ with probability one.

Extended states and quantum diffusion are expected to hold for the Anderson model for small $\lambda>0$ and for RBM with fixed large $\mathrm{W}$ in $3 \mathrm{D}$.

\section{Localization:}

$$
\left\langle\left|G\left(E_{\varepsilon} ; j, k\right)\right|^{2}\right\rangle \leq C_{\ell} \varepsilon^{-1} e^{-|j-k| / \ell(E)} .
$$

Note that the factor of $1 / \varepsilon$ on right hand side must be present because of (1.14) whenever $\left\langle\left|G\left(E_{\varepsilon} ; 0, k\right)\right|^{2}\right\rangle$ is summable. If (1.17) holds as $\varepsilon \rightarrow 0$ then there is an interval of dense pure point spectrum in a neighborhood of E. An alternate way to establish localization due to Aizenman and Molchanov [AM] is to prove that for some $\alpha, 0<\alpha<1$

$$
\left\langle\left|G\left(E_{\varepsilon} ; j, k\right)\right|^{\alpha}\right\rangle \leq C e^{-|j-k| / \ell(E, \alpha)}
$$

uniformly in $\varepsilon$.

1.4. Quantum Dynamics. There is a very natural dynamical interpretation of localization and extended states.

Define: $P(t, x) \equiv\left\langle\left|\delta_{x} \cdot e^{i t H} \delta_{0}\right|^{2}\right\rangle_{V}, x \in Z^{d}$, where $\mathrm{H}$ is the Anderson model and $\langle\cdot\rangle_{V}$ denotes the expectation over $\mathrm{V}$. We could also define $P$ for a RBM in infinite volume with $\mathrm{W}$ fixed. $\mathrm{P}(\mathrm{t}, \mathrm{x})$ is the probability of finding a particle at $\mathrm{x}$ at time $\mathrm{t}$ assuming it started at 0 . Note that by unitarity $\sum_{x} P(t, x)=1$ and the mean square displacement is defined by

$$
R^{2}(t) \equiv \sum_{x} P(t, x)|x|^{2}
$$

Roughly speaking we expect that time evolution has three basic forms:

Dynamical Localization: $R^{2}(t) \leq$ Const.

This holds if $|\lambda|$ is large or if the localization length $\ell(E)$ is uniformly bounded for all energies. For dynamical localization to hold more generally, the initial condition must be projected onto a region of spectrum where there is a uniform bound on $\ell(E)$.

\section{Quantum Diffusion: $R^{2}(t) \approx D t$}

Quantum diffusion is closely related to (1.16) and the existence absolutely continuous spectrum in $3 \mathrm{D}$, for $|\lambda| \leq 1$. The intuition for diffusion comes from thinking of a classical particle being weakly scattered by the potential or impurities as it moves on $\mathbb{Z}^{3}$. If we ignore memory effects, one might imagine that over long time scales the particle behaves like a random walk and diffuses. Although this classical picture is appealing it is probably harder to justify than its quantum counterpart. In the case of random Schrödinger, the best rigorous result about time evolution is is due to Erdös, Salmhofer and Yau [Er1] who establish diffusion for times $\lambda^{-2} \leq t \leq \lambda^{-(2+\varepsilon)}$ for some positive $\varepsilon$. At earlier times the motion is ballistic. For some results on quantum diffusion for RBM, see [Er3]. 


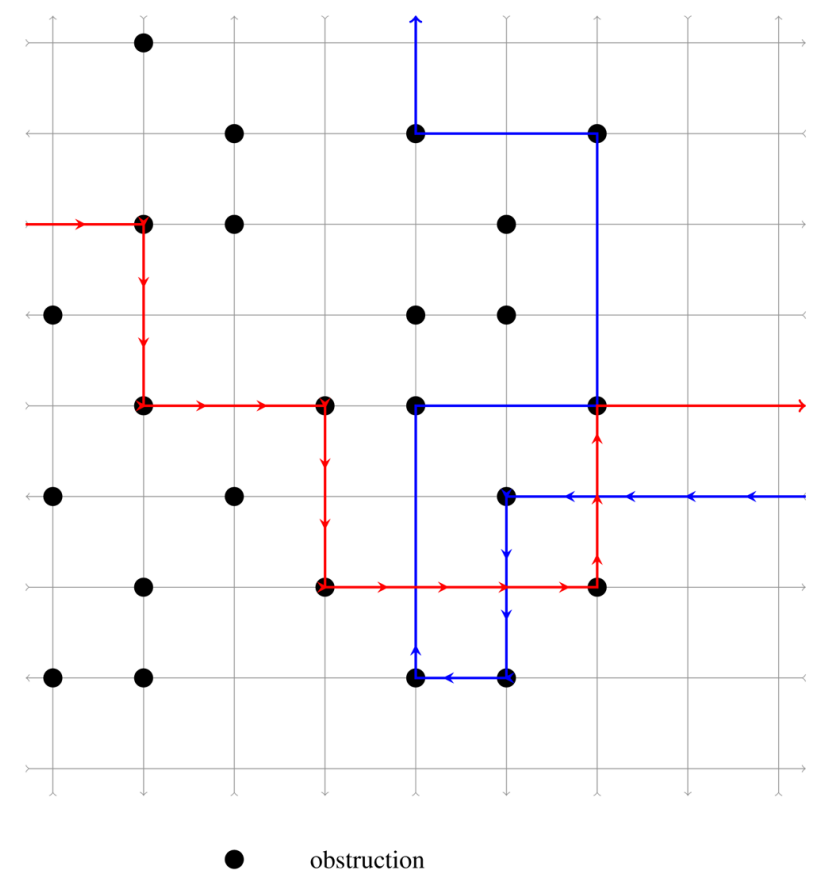

Figure 1. Orbits on Manhattan Lattice with obstructions

\section{Ballistic motion: $R^{2}(t) \approx D t^{2}$}

This can easily be established for $\lambda=0$ by Fourier analysis. In this case there is no scattering and classically the particle moves in a straight line. It should also hold for periodic potentials using a Bloch wave analysis. Note that small $\lambda$ is a singular pertubation since the time evolution is expected to change character from ballistic $\lambda=0$ to diffusive in 3D when $\lambda>0$ or localized in 1D and 2D.

Manhattan pinball: Next we describe a classical model of dynamics on the oriented Manhattan lattice with alternating orientations going north-south and east-west. See Figure 1. There are obstructions which appear independently on the vertices of the lattice with probability $\mathrm{p}$. We imagine a particle moving along the edges at unit speed following the orientations of the lattice. The particle may not pass through the obstruction and turns only when it encounters an obstruction. Thus the dynamics are determined by an initial edge and the location of the fixed randomly located obstructions.

This model was proposed and analyzed by Beamond, Owczarek and Cardy $[B O C]$ following related work of Gruzberg, Ludwig and Read [GLR]. Note that distinct orbits may pass through the same vertex but not the same edge.

Remarks: The Manhattan model looks classical but it is equivalent to a model of unitary quantum dynamics with $S U(2)$ edge disorder at a special energy. The phases arising from quantum mechanics are integrated out and in this case produce a positive measure. This is a quantum network model which arose from attempts to understand the Quantum Hall effect at the mobility edge. It belongs to the class C models in which time reversal symmetry is broken but Zeeman splitting is ignored. 
Theorem (Chalker) If $p>1 / 2$ all paths are closed with probability 1 .

The proof is based on results on bond percolation in $2 \mathrm{D}$. To see this draw an edge passing through each obstruction making $\pm 45^{\circ}$ with the axes and connecting the midpoints of the squares of the dual lattice. The edge orientation $\pm 45^{\circ}$ is selected to be plus if the vertical arrow passing through the obstruction is up and the horizontal arrow points right and minus for the down, right arrow. Such edges act as reflectors for an incoming particle. If $p>1 / 2$ these edges percolate and thus trap the particle.

It is also interesting to study this model on an infinite 1D cylinder of circumference L. It is obvious that for fixed $p>0$ the localization length is bounded by an exponential in $\mathrm{L}, p^{-L}$. However, this estimate is very crude and it is natural to conjecture that the localization length is less than $C_{p} L$. Such an estimate is also expected for the Anderson model. The linear dependence on $\mathrm{L}$ is known for spin systems such as the XY and Heisenberg model (see §1.6), using a simple deformation argument in [MS]. For the edge reinforced random walk a closely related estimate was proved in [MR2].

In 2D, a renormalization group argument by Beamond, Owczarek and Cardy ([BOC]) suggests that for any $0<p<1$, all paths are closed. Moreover, the average diameter of an orbit $\approx e^{c / p^{2}}$. This assertion is compatible with the prediction by Abrahams et al. [Abr] that all states are localized for the 2D Random Schrödinger model. At the moment there is no proof of localization for any value of $p$ with $0<p<1 / 2$.

There are related network models in $3 \mathrm{D},[\mathrm{OSC}]$. In this case one expects that there are diffusive orbits, and $\left\langle(X(t)-X(0))^{2}\right\rangle \approx D t$. For a recent review of the relation between classical and quantum models see Cardy [Car].

1.5. SUSY models and a History Dependent Random walk. In [Weg, SW] F. Wegner used the replica trick to study spectral properties of the Anderson model in terms of spin systems with hyperbolic symmetry. Following Wegner's work, K. Efetov introduced a class of supersymmetric "spin" models on the lattice which provided a mathematically precise dual representation for Green's functions. Efetov expresses expectations such as (1.16) as products of ratios of determinants of $E_{\varepsilon}-H$ using identities closely related to (1.11) and (1.12). For Hermitian RBM the spins are 4 by 4 matrices indexed by a lattice. They have both complex components in $\mathbb{C}$ and anti-commuting or Grassmann components which appear symmetrically. Efetov identified a non compact $S U(1 ; 1 \mid 2)$ symmetry which contains a hyperbolic $S U(1 ; 1)$ part as well as compact $S U(2)$ part arising from the Grassmann variables. The statistical weight of Efetov's model is invariant under this symmetry. Real symmetric matrices such as the Anderson model are described by another supersymmetric space parametrized by $8 \times 8$ matrices.

The 3D Efetov spin model is expected to have a phase transition from ordered to disordered phases of the spins which is equivalent to the Anderson transition described above. In statistical mechanics this transition is much like that of a magnetic bar whose spins are aligned at low temperature but when heated the spins are disordered and the bar no longer exhibits magnetization. More precisely if the spins in the supersymmetric Efetov model are ordered (aligned at low temperature), the corresponding normalized eigenvectors $v$ in a box of side $\mathrm{N}$ are extended: $\max _{j}|v(j)| \approx N^{-d / 2}$ as $\mathrm{N}$ gets large. In this case one expects quantum diffusion and the scaled eigenvalue spacing $\left(E_{k+1}-E_{k}\right) / \delta(E)$ should obey Wigner-Dyson 
statistics. On the other hand, when the spins are disordered, corresponding to high temperature, there is localization: the eigenstates decay exponentially fast about some lattice site. In this case, Poisson statistics describe the eigenvalue spacings. In $\S 1.6$ we review results about phase transitions for some classical models.

In practice, most theoretical physicists work with the nonlinear sigma model approximation to the Efetov model. In this case the matrix spins $s_{j}$ are constrained to lie on a manifold and in particular $s^{2}=I_{4}$. See $\S 3.3$ for a brief description. Thus it generalizes the well known Ising, XY and Heisenberg spins described later. Although this approximation is no longer the exact dual of a RBM, it preserves locality and symmetry and for most problems it is expected to give accurate and detailed information about the spectral theory of random matrices.

In 3 dimensions a proof of the ordered phase for the Efetov models has not been established. The key difficulty is due to the fact that weights on the spin configurations are not positive (oscillations and Grassmann variables) and there are gapless excitations responsible for diffusion. In the theoretical physics literature calculations are carried out perturbatively using spin wave and renormalization group analysis. However, in $\S 2.3$ we describe the Efetov model in one dimension where formulas are much simpler and correlations can be rigorously analyzed.

The $H^{2 \mid 2}$ model: In 1991 Zirnbauer [Zir2] introduced a simpler, supersymmetric spin system, which should have many features in common with of RBM and the Efetov model. We shall refer to it as the $\mathbf{H}^{2 \mid 2}$ model because the spins are vectors with values in the hyperbolic two plane augmented by two Grassmann fields. Zirnbauer established localization in one dimension. In three dimensions, [DSZ, DS] proved there is a phase transition from disorder to order. This transition is analogous to the conjectured Anderson transition. The $H^{2 \mid 2}$ model is defined in $\S 3$ and a sketch of the proof of the ordered phase is presented.

The Vertex Reinforced Jump Process: Although the $H^{2 \mid 2}$ model reflects many features expected to appear in quantum dynamics, it does not directly give spectral information about the Anderson model or RBM. However, recent results of Sabot and Tarres [ST] show that the $H^{2 \mid 2}$ model in d dimensions is equivalent to vertex reinforced jump process (VRJP) on $\mathbb{Z}^{d}$. This is a probabilistic, history dependent process in which a particle jumps to an adjacent vertex favoring those vertices at which it has spent more time. More precisely the VRJP is a continuous time process $X(t)$ where $\mathrm{X}$ jumps from the lattice site $\mathrm{j}$ to a neighbor $\mathrm{j}$ ' with rate $\beta\left(1+L_{j^{\prime}}(t)\right)$ where $L_{j^{\prime}}(t)$ is the amount of time it has spent at j' up to time t. The phase transition in 3D may be interpreted as follows: For weak reinforcement ( $\beta$ large) the particle has diffusive behavior, whereas for strong reinforcement ( $\beta$ small) the particle is exponentially localized near its starting point, $\operatorname{Prob}\{|X(t)-X(0)| \geq$ $r\} \leq C e^{-r / \ell}$. The parameter $\beta$ is proportional to the inverse temperature in the $H^{2 \mid 2}$ model. In two dimensions it is natural to expect that the long time dynamics is localized even for weak reinforcement. We have seen that quantum dynamics in two and three dimensions is expected to have similar properties. Sabot and Tarres $[\mathrm{ST}]$ also show that the discrete time linearly edge reinforced random walk is given by a modified $H^{2 \mid 2}$ model.

1.6. Statistical Mechanics and Mean Field Universality. We now describe some classical spin systems on a lattice in which the spins take values in a compact symmetric space $\mathcal{S}$. Well studied examples are $s_{j} \in \mathcal{S}=Z_{2}, \mathbb{S}^{1}, \mathbb{S}^{2}$ which are referred to as the Ising model, the XY model and the Heisenberg model. Here $\mathbb{S}^{m}$ 
denotes the m-dimensional sphere. In later sections we shall consider noncompact spaces

$$
\mathcal{S}=H^{2}=S U(1 ; 1) / U(1), \quad H^{2 \mid 2}, \quad U(1 ; 1 \mid 2) /(U(1 \mid 1) \times U(1 \mid 1))
$$

which parametrize the hyperbolic sigma model, the supersymmetric hyperbolic model and the (Hermitian) Efetov sigma model respectively. Spin systems whose targets are symmetric spaces or manifolds are often referred to as nonlinear sigma models. We shall see that the noncompact models are closely related to RBM.

The energy of a spin configuration $s_{j}, j \in Z^{d} \cap[-N, N]^{d} \equiv \Lambda_{N}$ in a periodic box of side $2 \mathrm{~N}$ is given by the nearest neighbor ferromagnetic interaction $\sum_{\left|j-j^{\prime}\right|=1}\left(s_{j}-\right.$ $\left.s_{j^{\prime}}\right)^{2}$ with a rotationally invariant expectation given by

$$
\langle A\rangle_{N}(\beta)=Z_{\Lambda_{N}}^{-1} \int A\left(\left\{s_{j}\right\}\right) e^{-\beta \sum_{\left|j-j^{\prime}\right|=1}\left(s_{j}-s_{j^{\prime}}\right)^{2}} \prod_{j \in \Lambda_{N}} d \mu\left(s_{j}\right) .
$$

Here $\beta>0$ is the inverse temperature and $d \mu\left(s_{j}\right)$ is the uniform measure on the sphere. The partition function $\mathrm{Z}$ is a normalization constant chosen so that $\langle 1\rangle_{N}(\beta)=1$.

For high temperature, $(\beta$ small $)$, the spins are disordered and correlations decay exponentially fast:

$$
0 \leq\left\langle s_{k} \cdot s_{j}\right\rangle_{N}(\beta) \leq C e^{-|k-j| / \ell(\beta)}, \quad|j-k| \leq N / 2
$$

where $\ell$ is the correlation length which is independent of N. Such estimates are relatively easy to prove for compact target spaces $\mathcal{S}$. This phase is analogous to localization for strong disorder.

Note that for large $\beta$, the spins tend to be locally aligned. In three dimensions the spins are ordered at long distances (uniformly in N) [FSS] and hence the magnetization, $\mathrm{M}$, is non zero:

$$
\left|\Lambda_{N}\right|^{-2} \sum_{j, k}\left\langle s_{j} \cdot s_{k}\right\rangle_{N}(\beta)=M_{N}^{2}(\beta) \geq\left[1-\frac{m+1}{\beta} G(0,0)\right]>0 .
$$

Here $G$ is the Green's function of the 3-dimensional lattice Laplacian. Note that $G(0,0)$ is finite in three dimensions but diverges in two dimensions. This corresponds to the fact that a random walk in two dimensions is recurrent whereas in $3 \mathrm{D}$ it is transient. If we then add a small external magnetic field in the 1 direction then the energy is defined to be

$$
\sum_{\left|j-j^{\prime}\right|=1}\left(s_{j}-s_{j^{\prime}}\right)^{2}-\varepsilon \sum_{j} s_{j}^{1} \quad \text { and } \quad M=\lim \varepsilon \downarrow 0\left\langle s_{0}^{1}\right\rangle(\beta, \varepsilon)
$$

where the expectation is defined in the infinite volume limit. If $M$ in (1.24) does not vanish in the limit $\varepsilon \rightarrow 0$ then we say that there is symmetry breaking since $\left\langle s_{0}^{1}\right\rangle(\beta, 0)=0$ by symmetry. The Mermin-Wagner theorem states that components of the spin $s^{a}, a \neq 1$ perpendicular to the external field satisfy the lower bound

$$
\sum_{j} e^{i j \cdot p}\left\langle s_{0}^{a} s_{j}^{a}\right\rangle(\beta, \varepsilon) \geq M^{2}\left(\beta p^{2}+\varepsilon M\right)^{-1}
$$

for $\left|p_{i}\right| \leq \pi$. This relation is valid in any dimension for systems with continuous symmetry and is proved using by integration by parts. In two dimensions this relation shows that $\mathrm{M}=0$ as $\varepsilon \rightarrow 0$. If not, the integral over $\mathrm{p}$ on the right side is divergent in $2 \mathrm{D}$ but the integral over the left side equals $\left(s_{0}^{a}\right)^{2} \leq 1$. The $1 / p^{2}$ 
indicates the presence of gapless modes called Goldstone modes which appear when there is continuous symmetry breaking as in (1.24) for $d \geq 3$. Note that in the noncompact case $\varepsilon>0$ is needed to make integrations well defined and is related to the imaginary part of the energy. There is some progress in extending MerminWagner type arguments to noncompact systems in [MR1, DMR].

In two dimensions, the XY model has the Kosterlitz-Thouless [KT] phase: slow power decay for large $\beta,\left\langle s_{0} \cdot s_{x}>(\beta) \approx|x|^{1 /(2 \pi \beta)}\right.$. An upper bound of this type for the XY was proved in [MS] and the lower bound in [FS1]. On the other hand, Polyakov [Pol] gave a renormalization argument which suggests that in the 2D Heisenberg model, spin correlations always decay exponentially fast with a correlation length $\ell(\beta) \approx e^{C \beta}$. Note the similarity to the localization length predicted for the two dimensional Anderson model, and RBM, [Abr] . In $\S 3.1$ we shall present arguments which suggest that the 2D $H^{2 \mid 2}$ model also has exponential decay for all $\beta$. A slightly more detailed discussion of spin systems appears in [Sp3].

The proof of the ordered phase (1.23) is based on reflection positivity, [FSS]. Unfortunately, this technique is not very flexible and it does not apply to the Efetov or $H^{2 \mid 2}$ models in 3D. The ordered phase for the $H^{2 \mid 2}$ model was proved in [DSZ] by using a family of identities which come from supersymmetry and by applying induction on length scales. A sketch of the proof is given in $\S 3$. T. Balaban [Bal] has developed another way to prove an ordered phase for systems with continuous symmetry via rigorous renormalization group techniques. The ordered phase for the Efetov sigma model in 3D is still open and is very closely related to the Anderson transition in 3D.

Next we formulate a mean field universality conjecture for the XY and Heisenberg models. Let $\Lambda_{N}$ be a periodic cube of side length $\mathrm{N}$ and define

$$
G_{N}(h, \beta)=\left\langle e^{h \cdot \sum_{\Lambda} S_{j} /|\Lambda|}\right\rangle_{N}(\beta) \quad \text { where } h \in R^{2} \text { or } R^{3} .
$$

Mean Field Universality Conjecture: In 3D,

$$
G_{N}(h, \beta) \rightarrow \int e^{M h \cdot S_{0}} d \mu\left(S_{0}\right)\left[1+O\left(\frac{1}{\beta N}\right)\right]
$$

as $N \rightarrow \infty$. The right side encodes the collective behavior of the spins by a single mean field spin, $S_{0}$. The spatial lattice structure has disappeared. The set of directions is determined by the orbit of a spin under the symmetry group of the interaction. Thus the leading term of (1.27) is governed by the 0 - mode. Note that $\beta$ only enters through the magnetization $M(\beta)$. Intuitively the right side is roughly obtained for large $\beta$ by supposing that all the spins are aligned in some direction $S_{0}$. To second order in h (1.27) is an identity but higher order terms in h require proof.

Remark: The leading term on the right side is roughly like the law of large numbers. We expect corrections of central limit type arising from the free Gaussian field fluctuations. These are crudely accounted for in error term $\left[1+O\left(\frac{1}{\beta N}\right)\right]$. Universality of means that the details of the interaction on the left are unimportant. In particular the spins need not have nearest neighbor interaction nor need they be restricted to the sphere. Once order is established, the right side is governed by the group invariance of the spin distribution. 
SUSY Motivation: The motivation for the above conjecture comes from SUSY statistical mechanics. The leading term is analogous to Wigner-Dyson local eigenvalue correlation (1.3) identified by [Ef1]. The finite volume correction formally was derived by Kravtsov and Mirlin [KM, Mir] by calculating the Gaussian fluctuations via spin wave theory. The leading term of the scaled local energy correlation (1.3) is corrected by the term $c g_{N}^{-2} \sin ^{2}(\pi \xi)$ where $g_{N}$ is the conductance $\approx \beta N^{d-2}$. In SUSY statistical mechanics, the role of $M(\beta)$ is played by the density of states $\rho(E)$. Note however there is an important difference between the SUSY and XY or Heisenberg case. $\mathrm{M}$ is an order parameter for classical spin systems but $\rho(E)$ is not an order parameter for SUSY models since it does not vanish in either the localized phase or diffusive phase. Moreover, in a large box $\rho(E)$ has very weak dependence on $\mathrm{N}$ (see $\S 2.2)$ but $M_{N}(1.23)$ has a power law dependence on $1 / \mathrm{N}$.

Jürg Fröhlich has recently explained a conceptual way to understand (1.27) by expressing the infinite volume expectation as a uniform superposition of pure states $<>_{u}(\beta)$ indexed by $u$ in the sphere $\mathbb{S}^{m}$. Thus for $m=2$ we have

$$
\begin{gathered}
G(h)=\int_{S^{2}}\left\langle e^{h \cdot \sum_{\Lambda} S_{j} /|\Lambda|}\right\rangle_{u}(\beta) d \mu(u)=\int_{S^{2}} e^{\left\langle h \cdot \sum_{\Lambda} S_{j} /|\Lambda|\right\rangle_{u}} d \mu(u)\left(1+O\left(\frac{1}{\beta N}\right)\right) \\
=\sinh (M|h|) / M|h|\left[1+O\left(\frac{1}{\beta N}\right)\right] .
\end{gathered}
$$

The exponent of the right hand side of (1.28) is given by $M h \cdot u$. The terms arising from the second cumulant go to zero because by infra-red bounds [FSS] the spin correlations $\left\langle S_{j} S_{k}\right\rangle_{u}-\left\langle S_{j}\right\rangle_{u}\left\langle S_{k}\right\rangle_{u}$ have a Fourier transform bounded by $\left(\beta p^{2}\right)^{-1}$ for $p \neq 0$ hence there is effectively a $1 / r$ decay in the spins producing an error term as above. For the XY model, the analog of (1.28) can be proved using a theorem of Fröhlich and Pfister $[\mathrm{FP}]$ which states that for almost all $\beta$ all states are superpositions of the form above. For more general symmetries such as those arising in SUSY with $U(1 ; 1 \mid 2)$ symmetry the expected formulas are similar. However, rigorous spin wave analysis is needed to control fluctuations about the 0 mode.

Acknowledgments. I would like thank the organizers for inviting me talk at the conference on Current Developments in Mathematics. I thank M. Disertori, M. Shamis, T. Shcherbina and W-M. Wang for helpful comments on an earlier draft of this review.

\section{SUSY statistical mechanics}

In this section we shall give an introduction to SUSY statistical mechanics and apply it to obtain detailed information about the density of states for GUE and also 3D band matrices. A recent theorem of T. Shcherbina will illustrate a form of mean field universality for 1D Gaussian RBM. In one dimension, Efetov's sigma model can be analyzed and localization is established with a localization length proportional to $\beta \approx W^{2} \rho(E)^{2}$. The origins of hyperbolic symmetry and a theorem about hyperbolic $\sigma$-model in $3 \mathrm{D}$ are explained in $\S 2.3$.

2.1. Gaussian and Grassmann integrals. The dual representation of the average of Green's function relies on averages of ratios of determinants. We first consider the inverse of the determinant expressed by a Gaussian integral identity. 
Let $z=\left(z_{1}, z_{2}, \ldots, z_{N}\right)$ with $z_{j}=x_{j}+i y_{j}$ denote an element of $\mathbb{C}^{N}$ and define the quadratic form

$$
[z ; H z]=\sum_{k, j} \bar{z}_{k} H_{k j} z_{j} .
$$

Recall $E_{\varepsilon}=E-i \varepsilon$. Then we can calculate the following Gaussian integrals:

$$
\int e^{-i\left[z ;\left(E_{\varepsilon}-H\right) z\right]} D z=(-i)^{N} \operatorname{det}\left(E_{\varepsilon}-H\right)^{-1} \quad \text { where } \quad D z \equiv \prod_{j}^{N} d x_{j} d y_{j} / \pi
$$

and

$$
\int e^{-i\left[z ;\left(E_{\varepsilon}-H\right) z\right]} z_{k} \bar{z}_{j} D z=(-i)^{N+1} \operatorname{det}\left(E_{\varepsilon}-H\right)^{-1} G\left(E_{\varepsilon} ; k, j\right) .
$$

$G\left(E_{\varepsilon}\right)$ is the Green's function (1.13). It is important to note that the integrals above are convergent provided that $\varepsilon>0$. The quadratic form $[z ;(E-H) z]$ is real so its contribution only oscillates. The factor of $i=\sqrt{-1}$ in the exponent is needed because the matrix $E-H$ has an indefinite signature when $\mathrm{E}$ is in the spectrum of H.

There is a similar identity in which the complex commuting variables $\mathrm{z}$ are replaced by anticommuting Grassmann variables $\psi_{j}, \bar{\psi}_{j}, \mathrm{j}=1,2 \ldots \mathrm{N}$ which satisfy

$$
\psi_{j} \psi_{k}=-\psi_{k} \psi_{j}, \quad \bar{\psi}_{j} \psi_{k}=-\psi_{k} \bar{\psi}_{j}, \quad \bar{\psi}_{j} \bar{\psi}_{k}=-\bar{\psi}_{k} \bar{\psi}_{j}, \quad \psi_{j}^{2}=\bar{\psi}_{j}^{2}=0 .
$$

Let $A$ be an $N \times N$ matrix and define

$$
[\psi ; A \psi]=\sum \bar{\psi}_{k} A_{k j} \psi_{j} \quad D \psi \equiv \prod_{j}^{N} d \bar{\psi}_{j} d \psi_{j}
$$

The integral of any polynomial in $\bar{\psi}, \psi$ with respect to $D \psi$ is algebraically defined to be equal to the coefficient of the top degree monomial written as $\prod^{N} \psi_{j} \bar{\psi}_{j}$. For example

$$
\int e^{-a[\psi ; \psi]} D \psi=\int \prod^{N}\left(1-a \bar{\psi}_{j} \psi_{j}\right) D \psi=\int \prod^{N}\left(1+a \psi_{j} \bar{\psi}_{j}\right) D \psi=a^{N} .
$$

The next two formulas are similar to those for the complex Gaussian integral

$$
\int e^{-[\psi ; A \psi]} D \psi=\operatorname{det} A, \quad \int \psi_{j} \bar{\psi}_{k} e^{-[\psi ; A \psi]} D \psi=\left(A^{-1}\right)_{j k} \operatorname{det} A .
$$

The bar superscript is just a label to denote a separate family of Grassmann variables. See [Ber, Mir, Sal, Sp3] for more details about Grassmann integration and its applications.

Main SUSY formulas: We shall see that Grassmann integration is extremely useful. It is used to cancel the unwanted determinant in (2.3) and enables us perform the average over the randomness in $H$. The basic SUSY (supersymmetric) representation for the Green's function follows from (2.3) and (2.4)

$$
G\left(E_{\varepsilon} ; k, j\right)=i \int e^{-i\left[z ;\left(E_{\varepsilon}-H\right) z\right]} e^{-i\left[\psi ;\left(E_{\varepsilon}-H\right) \psi\right]} z_{k} \bar{z}_{j} D z D \psi .
$$

This equation is the starting point for all SUSY formulas. Notice that if $\mathrm{H}$ has a Gaussian distribution, the expectation of (2.5) can be explicitly performed since $\mathrm{H}$ 
appears linearly. We obtain:

$$
\left\langle\operatorname{tr} G\left(E_{\varepsilon}\right)\right\rangle=i \int[z ; z] e^{-i E_{\varepsilon}([z ; z]+[\psi ; \psi])} e^{-\frac{1}{2}\left\langle\{[z ; H z]+[\psi ; H \psi]\}^{2}\right\rangle} D z D \psi .
$$

The resulting lattice field model will be quartic in the $z$ and $\psi$ fields. Notice that if the observable $i[z ; z]$ were absent from (2.6), then the determinants would cancel and the integral would be equal to 1 for all parameters. Thus in SUSY systems, the partition function is identically 1 . In a similar fashion we can obtain more complicated formulas for $\left\langle G\left(E_{\varepsilon} ; 0, j\right) \bar{G}\left(E_{\varepsilon} ; 0, j\right)>\right.$. To do this we must introduce additional variables $w \in \mathbb{C}^{N}$ and independent Grassmann variables $\chi, \bar{\chi}$ to obtain the second factor, $\bar{G}$, which is the complex conjugate of $\mathrm{G}$. These spin systems are much harder to analyze.

Now let $\mathrm{H}$ be an $N \times N$, GUE matrix. The average in (2.6) is calculated with

$$
<\{[z ; H z]+[\psi ; H \psi]\}^{2}>=\left\{[z ; z]^{2}+2[\psi ; z][z ; \psi]-[\psi ; \psi]^{2}\right\} / N .
$$

Proof of (1.10): If we omit the Grassmann variables

$$
(i)^{-N}<\operatorname{det}\left(E_{\varepsilon}-H\right)^{-1}>=<\int e^{-i\left[z ;\left(E_{\varepsilon}-H\right) z\right]} D z>=\int e^{-\left[z ; E_{\varepsilon} z\right]-[z ; z]^{2} / 2 N} D z .
$$

Let $r=[z, z]=\sum\left|z_{j}\right|^{2}$. Then we have

$$
<\operatorname{det}\left(E_{\varepsilon}-H\right)^{-1}>=C_{N} \int_{0}^{\infty} e^{-\frac{1}{2 N} r^{2}-i E_{\varepsilon} r} r^{N-1} d r
$$

After scaling $r \rightarrow N s$ we obtain an integral of the form

$$
\int_{0}^{\infty} e^{-N\left(s^{2} / 2-\ln s+i E_{\varepsilon} s\right)} d s / s .
$$

To calculate the average Green's function for GUE we shall apply the HubbardStratonovich identity which converts quartic expressions in $\mathrm{z}$ and $\psi$ in (2.7) into quadratic expressions we can explicitly calculate. Let $s_{1}, s_{2} \in \mathbb{R}$ and note that

$$
e^{-\frac{1}{2 N}[z ; z]^{2}}=c_{N} \int e^{-N s_{1}^{2} / 2} e^{i s_{1}[z ; z]} d s_{1}, \quad e^{\frac{1}{2 N}[\psi ; \psi]^{2}}=c_{N} \int e^{-N s_{2}^{2} / 2} e^{-s_{2}[\psi ; \psi]} d s_{2}
$$

where $c_{N}=\sqrt{N / 2 \pi}$. Using (2.7) and (2.8) we calculate the $\mathrm{N}$ dimensional integral (2.6) over $\psi, z$ in terms an integral over $s_{1}, s_{2}$. After integration by parts in $s_{1}$ we have

$$
\begin{gathered}
\frac{1}{N} \operatorname{tr}\left\langle G\left(E_{\varepsilon}\right)\right\rangle=\frac{i}{N} \int[z ; z] e^{-i E_{\varepsilon}([z ; z]+[\psi ; \psi])} e^{-\left\{[z ; z]^{2}+2[\psi ; z][z ; \psi]-[\psi ; \psi]^{2}\right\} / 2 N} D z D \psi=\left\langle s_{1}\right\rangle \\
\left\langle s_{1}\right\rangle \equiv c_{N}^{2} \int s_{1} e^{-N\left(s_{1}^{2}+s_{2}^{2}\right) / 2} e^{-i\left(E_{\varepsilon}-s_{1}\right)[z ; z]} e^{-i\left(E_{\varepsilon}-i s_{2}\right)[\psi ; \psi]}[1-[\psi ; z][z ; \psi] / N] d s_{1} d s_{2} D z D \psi \\
=c_{N}^{2} \int s_{1} e^{-N\left(s_{1}^{2}+s_{2}^{2}\right) / 2} \frac{\left(E_{\varepsilon}-i s_{2}\right)^{N}}{\left(E_{\varepsilon}-s_{1}\right)^{N}} R\left(s_{1}, s_{2}\right) d s_{1} d s_{2}
\end{gathered}
$$

where

$$
R=1-\left(E_{\varepsilon}-s_{1}\right)^{-1}\left(E_{\varepsilon}-i s_{2}\right)^{-1} .
$$

In (2.9) we have expanded $e^{-[\psi, z][z, \psi] / N}=1-[\psi, z][z, \psi] / N$. It produces $\mathrm{R}$ and is called the Fermion-Boson interaction. In this case it has a very simple form. 
There are 4 saddle points

$$
s_{1}^{* \pm}=E_{\varepsilon} / 2 \pm i \sqrt{1-\left(E_{\varepsilon} / 2\right)^{2}}, \quad s_{2}^{* \pm}=-i E_{\varepsilon} / 2 \pm \sqrt{1-\left(E_{\varepsilon} / 2\right)^{2}} .
$$

For $s_{1}$ we choose the saddle with positive imaginary part so that the pole of ( $E-$ $\left.i \varepsilon-s_{1}\right)^{-N}$ is not crossed. Note that $s^{*}=\left(s_{1}^{*+}, s_{2}^{*+}\right)$ is the dominant saddle because $R\left(s_{1}^{*+}, s_{2}^{*-}\right)=0$. We shift our contour of integration so that it passes through the two saddles $\left(s_{1}^{*+}, s_{2}^{* \pm}\right)$. Along this contour one checks that for $E$ satisfying $|E| \leq 1.8$, the maximum modulus of the integrand occurs at the saddle $s^{*}$. In particular, this deformation of contour avoids the small denominator $E_{\varepsilon}-s_{1}$ occurring when $s_{1} \approx E$. The Wigner semicircle law is given by

$$
\rho_{N}(E)=\frac{\operatorname{Im}}{N \pi} \operatorname{tr} G\left(E_{\varepsilon}\right)=\frac{1}{\pi} \operatorname{Im}<s_{1}>\approx \frac{1}{\pi} \operatorname{Im} s_{1}^{*} .
$$

A more detailed exposition of the calculations presented here can be found in [Dis]. See also [Sha] where the GOE case is calculated without using HubbardStratonovich. The main result for GUE is that for $|E| \leq 2-\delta, \delta>0$

$$
\rho_{N}(E)=\frac{1}{\pi} \sqrt{1-(E / 2)^{2}}+\frac{1}{N} O s c_{N}(E)+O\left(N^{-3 / 2}\right)
$$

where $\operatorname{Osc}_{N}(E)$ is a bounded but highly oscillatory contribution which can be explicitly computed. This contribution arises from a careful analysis of the second saddle. At the edges of the spectrum $E= \pm 2$ these saddle point analysis must be refined because the Hessian vanishes at the saddle so that the leading contribution is cubic. This gives rise to an Airy function instead of Wigner's semicircle. See [Dis].

2.2. Density of states for Gaussian RBM. The above results for GUE can also be carried out by using the classical methods of orthogonal polynomials. In fact, asymptotics of Hermite polynomials are obtained by integral representations much like those above. However, for Gaussian RBM orthogonal polynomial techniques do not seem to apply and pertubative or moment methods can only be controlled when $\varepsilon \geq W^{-1}$. The SUSY statistical mechanics techniques for Gaussian RBM are natural and estimates have little dependence on $\varepsilon \rightarrow 0$. On the other hand, they are not easy to apply for non-Gaussian distributions.

Let $\Lambda \subset \mathbb{Z}^{3}$ be a cube of side $\mathrm{N}$ with periodic boundary conditions. We shall assume that $\mathrm{J}$ in $(1.6)$ is

$$
J_{j k}=\left(-W^{2} \Delta_{\Lambda}+1\right)^{-1}(j, k) \approx C \frac{e^{-|j-k| / W}}{W^{2}|j-k|}
$$

where $\Delta_{\Lambda}$ is the lattice Laplacian with periodic boundary conditions. For a random band or GUE matrix the density of states is given by the limit as $N \rightarrow \infty$

$$
\rho_{N}(E)=\lim \varepsilon \downarrow 0 \frac{\operatorname{Im}}{\pi(N)^{d}}\left\langle\operatorname{tr}\left(E_{\varepsilon}-H\right)^{-1}\right\rangle .
$$

The average Green's function for Gaussian band matrices in three dimensions is a supersymmetric statistical mechanics model.

The analysis of random band matrices in [DPS] is in the same spirit as above except that we have many variables. Let $S=\left(S_{1}(j), S_{2}(j)\right) \in \mathbb{R}^{2}, j \in \Lambda \cap \mathbb{Z}^{d}$,

$$
\rho_{\Lambda}(E, \varepsilon) \equiv \frac{1}{|\Lambda|}\left\langle\operatorname{tr}(H-E-i \varepsilon)^{-1}\right\rangle_{R B M}=\left\langle S_{1}(0)\right\rangle_{S U S Y}
$$




$$
=C_{\Lambda} \int S_{1}(0) e^{-\sum_{j}\left[\mathbf{W}^{2}(\nabla S)(j)^{2}+S(j)^{2}\right] / 2} \cdot \mathbf{R} \cdot \prod_{j \in \Lambda} \frac{\left(i S_{2}(j)-E_{\varepsilon}\right)}{\left(S_{1}(j)-E_{\varepsilon}\right)} d S_{1}(j) d S_{2}(j) .
$$

The band width $\mathrm{W}$ is large but fixed and $\Lambda \uparrow \mathbb{Z}^{d}$ as in statistical mechanics. The Grassmann variables have all been integrated out and produce the Fermion-Boson factor coupling $S_{1}$ and $S_{2}$ given by

$$
\mathbf{R}=\operatorname{det}\left\{-W^{2} \Delta_{\Lambda}+1-\delta_{i j}\left(S_{1}(j)-E_{\varepsilon}\right)^{-1}\left(i S_{2}(j)-E_{\varepsilon}\right)^{-1}\right\} .
$$

For large $\mathrm{W}$ the spins are nearly equal and thus (2.15) is similar to the formula for GUE.

Although this formula looks rather complicated it can be analyzed rigorously as $\varepsilon \rightarrow 0$. We deform the contour of integration as we did for the GUE case. An essential feature of this model which makes it accessible to analysis is that the Hessian of the total action at the saddle point has a bounded inverse which decays exponentially. The cluster expansion [Sal] takes advantage of this fact and shows that spins at distances longer than $W$ are approximately independent. Thus estimates in a large box $\Lambda$ can essentially be reduced to a box of side W. Earlier work by Constantinescu et al. [Con] on Wegner's N orbital model obtained similar results also using SUSY statistical mechanics.

The main results of [DPS] are that for fixed $W \gg 1, \rho(E)$ is smooth and it has an asymptotic expansion in $W^{-1}$.

Theorem Let $\mathrm{d}=3,|E| \leq 1.8$ and $\mathrm{J}$ be given by (2.13). For $W \geq W_{0}, \rho(E)$ is smooth and the average Green's function for $\mathrm{RBM}<G\left(E_{\varepsilon}, j, j\right)>$ is uniformly bounded in $\varepsilon$ and $\Lambda$. It is approximately given by the semicircle distribution with corrections of order $1 / W^{2}$. Moreover, we have

$$
\left|\left\langle G\left(E_{\varepsilon} ; 0, x\right) G\left(E_{\varepsilon} ; x, 0\right)\right\rangle\right| \leq C e^{-m|x|}
$$

for $m \propto W^{-1}$.

Remark: One expects smoothness of the density of states for the Anderson model. However, this is a more difficult problem to analyze due to oscillatory contributions in SUSY produced by $\Delta$.

Remark: If we consider $<\left|G\left(E_{\varepsilon} ; j, k\right)\right|^{2}>$ given by (1.13) or averages such as $<\operatorname{det}^{2}(H-E)>$, we shall see that the Hessian has eigenvalues near 0 and there is slow decay of correlations which is related to quantum diffusion. This is also more difficult to control rigorously because of long range correlations.

2.3. Hyperbolic symmetry and $\left\langle\left|\operatorname{det}\left(E_{\varepsilon}-H_{\Lambda}\right)\right|^{-2}\right\rangle$. In this section we describe how hyperbolic symmetry arises and briefly describe results about hyperbolic spins systems on the lattice. We shall see that there are interesting new features due to the continuous symmetries that arise as $\varepsilon \rightarrow 0$. These symmetries can produce gapless modes in 3D called Goldstone modes. In this case, rather than a saddle point we have a saddle manifold.

In section 2.1 we derived a simple formula for the average of $\operatorname{det}\left(E_{\varepsilon}-H\right)^{-1}$ for the GUE distribution. We now study the expressions of the form $\left\langle\left|\operatorname{det}\left(E_{\varepsilon}-H\right)\right|^{-2}\right\rangle$ which are associated with hyperbolic symmetry. To make this symmetry clear let us consider the very simple case when $N=1$ and $H=h$ is a real Gaussian variable 
of unit variance. To simplify notation set $\mathrm{E}=0$, and use two complex variables $z, w$ to get the identity

$$
\begin{gathered}
\left\langle\left|\operatorname{det}\left(E_{\varepsilon}-H\right)\right|^{-2}\right\rangle=\left\langle\left|\left(E_{\varepsilon}-h\right)\right|^{-2}\right\rangle=\frac{1}{\pi^{2}} \int\left\langle\exp \left[i h\left(z^{*} z-w^{*} w\right)-\varepsilon\left(z^{*} z+w^{*} w\right)\right]\right\rangle d z d w \\
=\frac{1}{\pi^{2}} \int \exp \left[-\frac{1}{2}\left(z^{*} z-w^{*} w\right)^{2}-\varepsilon\left(z^{*} z+w^{*} w\right)\right] d z d w \approx C \varepsilon^{-1} .
\end{gathered}
$$

Note that $\varepsilon$ breaks the hyperbolic symmetry so that the integral is well defined. If we had no absolute value, we would get $\left(z^{*} z+w^{*} w\right)^{2}$ and hence (2.18) would be convergent even as $\varepsilon \rightarrow 0$.

In three dimensions, the hyperbolic sigma model was analyzed in [SZ]. The spins $h_{j}$ take values in a hyperboloid $h_{j}=\left(x_{j}, y_{j}, z_{j}\right)$ which satisfies the constraint $z_{j}^{2}-x_{j}^{2}-y_{j}^{2}=1$ with $z>0$. The nearest neighbor Gibbs weight is proportional to

$$
e^{-\beta \sum_{j, j^{\prime} \in \Lambda} h_{j} \cdot h_{j^{\prime}}-\varepsilon \sum_{j} z_{j}}
$$

where $h \cdot h^{\prime}=z z^{\prime}-x x^{\prime}-y y^{\prime}$ and the sum above ranges over nearest neighbor $j, j^{\prime}$ in a $3 \mathrm{D}$ periodic box $\Lambda$. This model describes $\left\langle\left|\operatorname{det}\left(E_{\varepsilon}-H_{\Lambda}\right)\right|^{-2}\right\rangle$ where $\mathrm{H}$ is a $3 \mathrm{D}$ $\mathrm{RBM}$ in the sigma model approximation. It is a special case of Wegner's hyperbolic sigma model.

The analysis of the hyperbolic sigma model relies on the horospherical parametrization of the hyperboloid:

$$
z=\cosh t+s^{2} e^{t} / 2, \quad x=\sinh t-s^{2} e^{t} / 2, \quad y=s e^{t}
$$

where $s, t \in \mathbb{R}$. This parametrization is a consequence of the Iwasawa decomposition. It plays a crucial role here and in the $H^{2 \mid 2}$ model in $\S 3$. The Gibbs weight now has the form $e^{-A(s, t)} \prod e^{t_{j}} d s_{j} d t_{j}$ where

$A(s, t)=\beta \sum_{j \sim j^{\prime} \in \Lambda}\left[\cosh \left(t_{j}-t_{j^{\prime}}\right)+\frac{1}{2}\left(s_{j}-s_{j^{\prime}}\right)^{2} e^{\left(t_{j}+t_{j^{\prime}}\right)}\right]+\varepsilon \sum_{j \in \Lambda}\left[\cosh \left(t_{j}\right)+\frac{1}{2} s^{2} e^{t_{j}}\right]$.

The advantage of these coordinates is that the action is quadratic in the $s_{j}$ variables. In fact we shall see in $\S 3.1$ that the quadratic form in $s$ is the generator of a random walk in a random environment with local conductance $e^{t_{j}+t_{j^{\prime}}}$ across each edge $j, j^{\prime}$ of the lattice. After integrating out the $s_{j}$ we get an effective action of $t_{j}$ which is convex for all $\beta, \varepsilon$, thus there is no phase transition for this sigma-model. D. Brydges has given a simple argument for convexity based on the matrix tree theorem. Hence the Brascamp-Lieb [BL] inequalities can be applied. The Hessian is bounded below as a quadratic form by $-\beta \Delta$. Let $G_{0}=(-\beta \Delta+\varepsilon)^{-1}$.

Theorem ([SZ]) In the three dimensional hyperbolic sigma model all moments of the form $<\cosh ^{p}\left(t_{0}\right)>$ are bounded for all $\beta$. The estimates are uniform in $\varepsilon$ provided we first take the limit $\Lambda \rightarrow \mathbb{Z}^{3}$. Moreover there is a constant $\mathrm{C}$ such that the spin correlations satisfy

$$
\frac{1}{C}\left[f ; G_{0} f\right] \leq \sum_{j, k} f(j) f(k)\left\langle y_{j} y_{k}\right\rangle(\beta, \varepsilon) \leq C\left[f ; G_{0} f\right]
$$

where $f(j) \geq 0$ is any function with rapid decay. This estimate indicates that correlations $\left\langle y_{j} y_{k}\right\rangle(\beta, \varepsilon)$ have a slow decay $\approx|j-k|^{-1}$ matching the Green's function of $-\Delta$ in $3 \mathrm{D}$. See [Sp3] for a slight improvement of the results of [SZ]. 
2.4. Efetov's SUSY $\sigma$-model in one dimension. Efetov's supersymmetric sigma model with $S U(1,1 \mid 2)$ is used to describe many quantum systems with disorder and is widely studied in theoretical physics. The sigma model is an approximation to the exact SUSY dual statistical mechanics model which describes RBM. The sigma approximation preserves locality and the symmetry of the exact model. It is expected to describe all the qualitative features of RBM in $3 \mathrm{D}$ and as well as $1 \mathrm{D}$ and $2 \mathrm{D}$ systems. In 3D this model seems to be well beyond the reach of present rigorous mathematical techniques.

However, in one dimension the Efetov sigma model has a very appealing form which we describe below. The Grassmann variables can be explicitly integrated out and the resulting model is a classical Heisenberg coupled to a hyperbolic sigma model which can be simply analyzed. In [Ef3] Efetov derived the following expression for a 1D sigma model describing the conductance in a chain of length $\mathrm{L}$. It is a nearest neighbor spin model with positive weights given as follows. Let $h_{j}$ and $\sigma_{j}$ take values in a hyperboloid and the sphere $S^{2}$ respectively, see $\S 1.6, \S 2.3$. The Gibbs weight is then proportional to

$$
\prod_{j=0}^{L}\left(h_{j} \cdot h_{j+1}+\sigma_{j} \cdot \sigma_{j+1}\right) e^{\beta\left(\sigma_{j} \cdot \sigma_{j+1}-h_{j} \cdot h_{j+1}\right)} .
$$

To make the integral well defined we fix each of the spins $h_{0}, \sigma_{0}, h_{L}, \sigma_{L}$ equal to $(0,0,1)$. Physically this corresponds to placing leads at the end of the chain to measure its conductance. As in classical statistical mechanics, the parameter $\beta>0$ is referred to as the inverse temperature and $\beta \approx W^{2} \rho(E)^{2}$ where $\rho(E)$ is the density of states, and $\mathrm{W}$ is the band width. Both the compact and noncompact symmetries are apparent. The Fermion-Boson coupling is just given by the first factor in the product above. In higher dimensions, integrating out the Grassmann variables leads to much more complicated expressions.

In $1 \mathrm{D}$ when $\beta \gg 1$, the spins are aligned provided $L \ll \beta \approx \rho^{2} W^{2}$. At longer lengths $L$ the spins are disordered and the conductance of the chain goes to 0 , as $e^{-c L / \beta}$. Once the explicit integration over the Grassmann variables is performed, the proof of these statements is straightforward and will appear in joint work with Disertori following ideas of M. Zirnbauer.

Remark: We believe that similar results hold without the sigma approximation. This would imply localization for $1 \mathrm{D} \mathrm{RBM}$ with $\ell(E) \approx W^{2} \rho(E)^{2}$.

2.5. Average of Determinants and the Heisenberg model. In this section we consider Gaussian random band matrices $\mathrm{H}$ with covariance $J_{j, k}$ given by (2.13) in a periodic box of side N. This is often referred to as the Fermion-Fermion sector. Define

$$
F_{N}\left(E, E^{\prime}\right)=\left\langle\operatorname{det}(H-E) \operatorname{det}\left(H-E^{\prime}\right)\right\rangle .
$$

We shall use Grassmann integration and duality to show that this expression is closely related to the Heisenberg model described in $\S 1.6$. The sphere parametrizes the zero modes. Let $\bar{\psi}_{j}, \psi_{j}, \bar{\chi}_{j}, \chi_{j}$ denote Grassmann variables indexed by $j \in \Lambda_{N}$. Then using (2.4) we have

$$
\operatorname{det}(H-E) \operatorname{det}\left(H-E^{\prime}\right)=\int e^{-[\bar{\psi} ;(H-E) \psi]-\left[\bar{\chi}\left(H-E^{\prime}\right) \chi\right]} D \psi D \chi .
$$


Let $M_{j}$ be defined by

$$
M_{j}=\left(\begin{array}{ll}
\bar{\psi}_{j} \psi_{j} & \bar{\psi}_{j} \chi_{j} \\
\bar{\chi}_{j} \psi_{j} & \bar{\chi}_{j} \chi_{j}
\end{array}\right)
$$

and set $\tilde{E}=\operatorname{diag}\left(E, E^{\prime}\right)$. Next we average over the H. Since H is Gaussian and has covariance $J_{j k}$ we get

$$
\left\langle\operatorname{det}(H-E) \operatorname{det}\left(H-E^{\prime}\right)\right\rangle=\int e^{-\sum J_{j, k} \operatorname{Tr}\left(M_{j} M_{k}\right)+\sum_{j} \operatorname{Tr}\left(M_{j} \tilde{E}\right)} D \psi D \chi .
$$

Now we use Hubbard-Stratonovich and let $X_{j}$ be $2 \times 2$ Gaussian Hermitian matrices such that

$$
\left\langle e^{+i T r\left(Y_{j} X_{j}\right)}\right\rangle_{X}=e^{-\sum J_{j k} \operatorname{Tr}\left(Y_{j} Y_{k}\right)}
$$

where the subscript is the average over $\mathrm{X}$. Then we have

$$
F_{N}\left(E, E^{\prime}\right)=\int\left\langle e^{\sum_{j} \operatorname{tr}\left(i X_{j}+\tilde{E}\right) M_{j}}\right\rangle_{X} D \psi D \chi .
$$

The integral over the $\mathrm{M}$ in (2.25) can be computed since the Grassmann variables appear quadratically and factor. Applying (2.4) we get

$$
F_{N}\left(E, E^{\prime}\right)=\left\langle\prod_{j} \operatorname{det}\left(i X_{j}+\tilde{E}\right)\right\rangle_{X}=\left\langle(-1)^{N} \prod_{j} \operatorname{det}\left(X_{j}-i \tilde{E}\right)\right\rangle_{X} .
$$

By (2.13) the Gibbs weight for the Gaussian measure in $\mathrm{X}$ is proportional to

$$
e^{-\frac{1}{2} \operatorname{Tr} \sum_{j}\left[W^{2}(\nabla X)_{j}^{2}+X_{j}^{2}\right]} \prod D X_{j}
$$

where DX is the Lebesgue measure on two by two Hermitian matrices.

Since $\mathrm{W}$ is assumed to be large, the $X_{j}$ typically depend slowly on $j$. Hence it is natural to look for a constant saddle point (manifold) of the action and consider fluctuations about it. The saddle point satisfies the following equation:

$$
\partial / \partial X\left\{\operatorname{Tr}\left[-X^{2} / 2+\log (X-i \tilde{E})\right]\right\}=0 \text { thus } \quad X^{2}-i \tilde{E} X-I_{2}=0
$$

where $I_{2}=\operatorname{diag}(1,1)$. Let $\mathrm{E}=\mathrm{E}$ '. Then the saddle point $X_{s}$ has the following form:

$$
\check{X}_{s}=\{i E / 2 \pm \bar{\rho}(E)\} I_{2} \quad \text { or } \quad \hat{X}_{s}=i E / 2 I_{2} \pm \bar{\rho}(E) \sigma_{3}
$$

where

$$
\bar{\rho}(E)=\pi \rho(E)=\sqrt{1-(E / 2)^{2}}
$$

and $\sigma_{3}=\operatorname{diag}(1,-1)$. Note that $U^{*} \hat{X}_{s} U$ is also a critical point for any unitary $U$ and solves (2.28). The orbit forms a saddle manifold isomorphic to the sphere.

We claim that the dominant contribution is near $\hat{X}_{s}$. To see this, consider fluctuations about a saddle $X_{s}, X_{j}=X_{s}+Y_{j}$ where $Y_{j}$ is a small 2 by 2 Hermitian matrix. In terms of $Y$, the action is

$$
\frac{1}{2} \operatorname{Tr} \sum_{j}\left[W^{2}(\nabla Y)_{j}^{2}+\left(Y_{j}+X_{s}\right)^{2}\right]-\sum_{j} \ln \operatorname{det}\left(Y_{j}+X_{s}-i E I_{2}\right) .
$$

Note that if $\bar{X}$ is the complex conjugate of $X$,

$$
X_{s}-i E I_{2}=\bar{X}_{s} \quad \text { and } \quad \bar{X}_{s}^{-1}=X_{s}
$$


The linear terms cancel since we are at a saddle point and the contribution of the constant terms have equal modulus. The quadratic contribution in $\mathrm{Y}$ is

$$
\frac{1}{2} \operatorname{Tr}\left[W^{2}(\nabla Y)_{j}^{2}+\left(Y_{j}^{2}\right)-\left(X_{s} Y_{j}\right)^{2}\right] .
$$

If $X_{s}=\check{X}_{s}$ then

$$
\operatorname{Tr}\left[\left(Y_{j}^{2}\right)-\left(X_{s} Y_{j}\right)\right]^{2}=\operatorname{Tr} Y^{2}\left[1-\{i E / 2 \pm \bar{\rho}(E)\}^{2}\right]
$$

Note that real part of the right side is definite for $|E|<2$. However, if $X_{s}=\hat{X}_{s}$ then the off diagonal elements of $\mathrm{Y}$ cancel:

$$
\operatorname{Tr}\left[\left(Y_{j}^{2}\right)-\left(\hat{X}_{s} Y_{j}\right)^{2}\right]=y_{11}^{2}\left(1-\{i E / 2+\bar{\rho}(E)\}^{2}\right)+y_{22}^{2}\left(1-\{i E / 2-\bar{\rho}(E)\}^{2}\right)
$$

In conclusion, although the two saddles have equal contributions in modulus, the Hessian about the second saddle produces the dominant contribution because of the coefficient of the variables $\left|y_{12}\right|^{2}$ vanishes. This means that the saddle manifold, $\mathbb{S}^{2}$, should govern the large $\mathrm{W}$ behavior. Since the coefficient $\left|y_{12}\right|^{2}$ vanishes of our Hessian has two (zero) Goldstone modes corresponding to the dimension of $\mathbb{S}^{2}$.

In the sigma model approximation we fix the eigenvalues of X. Thus for $U_{j} \in$ $S U(2)$ we have

$$
X_{j}=i E / 2 I_{2}+\bar{\rho}(E) U_{j}^{*} \sigma_{3} U_{j} \equiv i E / 2 I_{2}+\bar{\rho}(E) S_{j} .
$$

In this approximation we get the Heisenberg model described in $\$ 1.6$ at inverse temperature $\beta=W^{2} \bar{\rho}^{2}$. If the Heisenberg model is ordered then one might expect that the saddle manifold produces the main contribution to $F_{N}$ in (2.26). If we substitute $(2.30)$ into $(2.26)$ and let $\tilde{E}=\operatorname{diag}\left(E, E^{\prime}\right)=E I_{2}+\xi / \rho N^{-d} \sigma_{3}, \quad e_{3}=$ $(0,0,1)$ we get

$$
\frac{F_{N}\left(E+\xi / \rho N^{d}, E-\xi / \rho N^{d}\right)}{F_{N}(E, E)} \approx\left\langle e^{2 i \pi \xi N^{-d} \sum_{j} e_{3} \cdot S_{j}}\right\rangle_{N}(\beta) .
$$

The expression on the right should be compared to (1.28).

The informal discussion above is made precise in $1 \mathrm{D}$ for RBM by a theorem of T. Shchberina.

Theorem ([Shc2]). Let $\mathrm{H}$ denote a Gaussian RBM with covariance given by (2.13). Let $F_{N}$ be defined by (2.24). In one dimension, for $W^{2} \gg N$ and $|E|<2$

$$
\frac{F_{N}(E+\xi / \rho N, E-\xi / \rho N)}{F_{N}(E, E)} \rightarrow \frac{\sin (2 \pi \xi)}{2 \pi \xi} \text { as } \quad N \rightarrow \infty .
$$

Remarks: The theorem also holds for GUE matrices, hence it illustrates the validity of mean field theory discussed in $§ 1.6$. In one dimension the Heisenberg model is ordered at distances less than $\beta \approx \rho^{2} W^{2}$. However, if $N \gg W^{2}$ the spins are independent at long distances in $1 \mathrm{D}$ so the left side should go to 1 . In three dimensions, it is natural to conjecture that (2.31) holds for fixed large $\mathrm{W}$ as $\mathrm{N}$ gets large since the 3D Heisenberg model is ordered at all distances, [FSS]. More general products of $2 \mathrm{~m}$ determinants of Wigner matrices were studied in [Shc1]. For $m \geq 2$ the expectation of product is close to that for GUE except there are simple corrections arising from the fourth cumulant of the distribution.

Remark: In [Shc3], Shcherbina has recently applied related arguments to a more complicated SUSY model to prove universality of the pair correlation $\rho_{2}$ for RBM with any finite number of blocks. 


\section{A phase transition for the $H^{2 \mid 2}$ model}

In this section we describe results for a simpler version of the supersymmetric Efetov models which was introduced by Zirnbauer in (1991), [Zir2]. This is a system of interacting spins indexed by $\mathbb{Z}^{d}$. We call this model the $H^{2 \mid 2}$ model since the spins have two real components in a hyperboloid and two Grassmann partners to make it supersymmetric. It is expected to qualitatively reflect the phenomenology of Anderson's model described in $\S 1.2$.

The $H^{2 \mid 2}$ is related to the hyperbolic sigma model discussed in $\S 2.3$ but the additional Grassmann variables change the character of the model. A key feature of the $H^{2 \mid 2}$ model is that in horospherical coordinates the Grassmann degrees of freedom can be explicitly integrated out to produce a real effective action with positive weights. Thus probabilistic methods can be applied. In fact we will show that correlations in the model are expressed as a random walk in a correlated random environment.

In three or more dimensions, [DSZ, DS] proved that the $H^{2 \mid 2}$ model has a phase transition which is analogous to the Anderson transition. In the ordered phase $\beta \gg 1$, there are gapless modes and diffusion. For $0<\beta \ll 1$ there is exponential localization. The analysis of the phase transition relies heavily on identities arising from symmetries and on the study of a random walk in a strongly correlated random environment, see (3.5) below. The generator of this walk is not uniformly elliptic. If it were, we would always be in a diffusive phase. At low temperature in $3 \mathrm{D}$ we must control fluctuations of the environment. These are controlled by proving inductively that fluctuations of the local conductance, are bounded at successively longer length scales. Once strong estimates on these fluctuations are proved, diffusion follows since then our generator is effectively elliptic. When $0<\beta \ll 1$ the effective conductance goes to 0 .

As mentioned in $\S 1.5$, Sabot and Tarres proved that the $H^{2 \mid 2}$ model is equivalent to a history dependent process on $\mathbb{Z}^{d}$ called the vertex reinforced jump process (VRJP). In this process the particle jumps to an adjacent site favoring those where it has spent more time. The transition in 3D can be described as a transition from localization and recurrence (strong reinforcement, high temperature) to diffusion and transience for weak reinforcement. In two dimensions we conjecture that the walk does not diffuse even when the reinforcement is weak. As in the Anderson model or Heisenberg model we conjecture that the walk localizes about its initial point with a localization length of $e^{c \beta}$ where $\beta$ is inversely proportional to the magnitude of the reinforcement. See $\S 3.1$ for additional comments.

3.1. Definition of the model and Theorems. In order to define the $H^{2 \mid 2}$ sigma model, let $u_{j}$ be a vector at each lattice point $j \in \Lambda \subset \mathbb{Z}^{d}$ with three real components and two Grassmann components $u_{j}=\left(z_{j}, x_{j}, y_{j}, \xi_{j}, \eta_{j}\right)$, where $\xi, \eta$ are odd elements and $z, x, y$ are even elements of a real Grassmann algebra. The scalar product is defined by

$$
\left(u, u^{\prime}\right)=-z z^{\prime}+x x^{\prime}+y y^{\prime}+\xi \eta^{\prime}-\eta \xi^{\prime}, \quad(u, u)=-z^{2}+x^{2}+y^{2}+2 \xi \eta
$$

and the action is obtained by summing over nearest neighbors $j, j^{\prime}$

$$
\mathcal{A}[u]=\frac{1}{2} \sum_{\left(j, j^{\prime}\right) \in \Lambda} \beta\left(u_{j}-u_{j^{\prime}}, u_{j}-u_{j^{\prime}}\right)+\sum_{j \in \Lambda} \varepsilon_{j}\left(z_{j}-1\right) .
$$


The sigma model constraint, $\left(u_{j}, u_{j}\right)=-1$, is imposed so that the field lies on the SUSY hyperboloid, $H^{2 \mid 2}$.

We choose the branch of the hyperboloid so that $z_{j} \geq 1$ for each $j$. It is very useful to parametrize this manifold in horospherical coordinates:

$$
x=\sinh t-e^{t}\left(\frac{1}{2} s^{2}+\bar{\psi} \psi\right), \quad y=s e^{t}, \quad \xi=\bar{\psi} e^{t}, \quad \eta=\psi e^{t},
$$

and

$$
z=\cosh t+e^{t}\left(\frac{1}{2} s^{2}+\bar{\psi} \psi\right)
$$

where $t$ and $s$ are real and $\bar{\psi}, \psi$ are odd elements of a real Grassmann algebra.

In these coordinates, the weight of a field or spin configulation is $e^{-\mathcal{A}}$ where the sigma model action $\mathcal{A}$ is given by

$$
\begin{gathered}
\mathcal{A}[t, s, \psi, \bar{\psi}]=\sum_{(i j) \in \Lambda} \beta\left(\cosh \left(t_{i}-t_{j}\right)-1\right) \\
+\frac{1}{2}\left[s ; D_{\beta, \varepsilon} s\right]+\left[\bar{\psi} ; D_{\beta, \varepsilon} \psi\right]+\sum_{j \in \Lambda} \varepsilon_{j}\left(\cosh t_{j}-1\right) .
\end{gathered}
$$

Here $D_{\beta, \varepsilon}=D_{\beta, \varepsilon}(t)$ is the generator of a random walk in random environment, given by the quadratic form

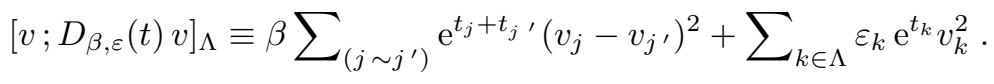

If $t_{j}=0$ then $\mathrm{D}$ is the lattice Laplacian. Note that the action is quadratic in the Grassmann and $\mathrm{s}$ variables. We define the corresponding expectation by $<\cdot>=$ $<\cdot>_{\Lambda, \beta, \varepsilon}$.

The weights, $e^{t_{j}+t_{j^{\prime}}}$ in (3.5), are the local conductances across a nearest neighbor edge $j, j^{\prime}$. The $\varepsilon_{j} e^{t_{j}}$ term is a killing rate for the walk at $j$. For the random walk starting at 0 without killing, we take $\varepsilon_{0}=1$ and $\varepsilon_{j}=0$ otherwise. If we set $\varepsilon_{j}=\varepsilon$ then $\varepsilon$ is analogous to the imaginary part of the energy in RBM.

After integrating over the Grassmann variables $\psi, \bar{\psi}$ and the variables $s_{j} \in \mathbb{R}$ we get the effective field theory with action $\mathcal{E}_{\beta, \varepsilon}(t)$ and partition function

$$
Z_{\Lambda}(\beta, \varepsilon)=\int e^{-\mathcal{E}_{\beta, \varepsilon}(t)} \prod e^{-t_{j}} d t_{j} \equiv \int e^{-\beta \mathcal{L}(t)} \cdot\left[\operatorname{det} D_{\beta, \varepsilon}(t)\right]^{1 / 2} \prod_{j} e^{-t_{j}} \frac{d t_{j}}{\sqrt{2} \pi} .
$$

where

$$
\mathcal{L}(t)=\sum_{j \sim j^{\prime}}\left[\cosh \left(t_{j}-t_{j^{\prime}}\right)-1\right]+\sum_{j} \frac{\varepsilon_{j}}{\beta}\left[\left(\cosh \left(t_{j}\right)-1\right] .\right.
$$

Note that the determinant is a positive but nonlocal functional of the $t_{j}$ hence the effective action, $\mathcal{E}=\mathcal{L}-1 / 2 \ln \operatorname{Det} D_{\beta, \varepsilon}$, is also nonlocal. By the matrix tree theorem $\ln \operatorname{Det} D_{\beta, \varepsilon}(t)$ is convex as a function of t. Thus $\mathcal{L}$ and $\ln \operatorname{Det} D$ compete. The additional factor of $e^{-t_{j}}$ arises from a Jacobian. Because of the internal supersymmetry, we know that for all values of $\beta, \varepsilon$ the partition function $Z(\beta, \varepsilon) \equiv 1$. This identity holds even if $\beta$ is edge dependent, $\beta_{e}$. Some of the Ward identities needed for our analysis can be obtained by taking derivatives in $\beta_{e}$. 
For large $\beta$ the value of $\left\{t_{j}\right\}$ which maximizes the integrand is a constant $t^{*}$ given by appendix in [DSZ]

$$
\begin{gathered}
1-e^{2 t^{*}}=\left(-\beta \Delta+\varepsilon e^{-t^{*}}\right)^{-1}(0,0), \\
\text { 1D: } \varepsilon \mathrm{e}^{-t^{*}} \simeq \beta^{-1}, \quad 2 \mathrm{D}: \varepsilon \mathrm{e}^{-t^{*}} \simeq \mathrm{e}^{-\beta}, \quad 3 \mathrm{D}: t^{*} \approx 0 .
\end{gathered}
$$

In one and two dimensions $(0<\varepsilon \ll 1)$ large negative values of $t$ are favored and the sensitive dependence of $t^{*}$ on $\varepsilon$ suggests disorder and localization. On the other hand in $3 \mathrm{D}$ order should be present for large $\beta$. For small values of $\beta, t^{*}$ has sensitive dependence on $\varepsilon$ for all dimensions. Although it is reasonable to expect that $t^{*}$ gives a reasonable approximation to the behavior of $\mathrm{t}$, note that one must be careful about interpreting it due large deviations of the $t$ field. For example, by a Ward identity we know that $\left\langle e^{t_{j}}\right\rangle=1$ for all $\beta$, $\varepsilon$. In $1 \mathrm{D}$ and 2D, $<e^{t_{j}+t_{j^{\prime}}}>$ is expected to diverge, whereas $\left\langle e^{t_{j} / 2}>\right.$ should become small as $\varepsilon \rightarrow 0$. One way to adapt the saddle approximation so that it is sensitive to different observables is to include the observable when computing the saddle point. For example, when taking the expectation of $e^{p t_{0}}$, the saddle is only slightly changed when $p=1 / 2$ but for $p=2$ it will give a divergent contribution when there is localization.

The analog of the Green's function $<\left|G\left(E_{\varepsilon} ; 0, x\right)\right|^{2}>$ of the Anderson model is given by

$$
<y_{0} y_{x}>(\beta, \varepsilon)=<s_{0} e^{t_{0}} s_{x} e^{t_{x}}>(\beta, \varepsilon)=<e^{\left(t_{0}+t_{x}\right)} D_{\beta, \varepsilon}(t)^{-1}(0, x)>(\beta, \varepsilon)
$$

where we have used (3.3) and (3.4) to obtain these identities. This formula expresses correlations for the $H^{2 / 2}$ model as a random walk in a random environment. If $\left\{t_{j}=\right.$ $0\}$ then we see from (3.5), that (3.8) will have diffusive behavior. The expectation also has a Schrödinger representation since

$$
e^{-t} D_{\beta, \varepsilon}(t) e^{-t}=-\beta \Delta+\beta V(t)+\varepsilon e^{-t_{j}}, \quad V_{j}(t)=\sum_{i:|i-j|=1}\left(\mathrm{e}^{t_{i}-t_{j}}-1\right) .
$$

In one and two dimensions the value of $t^{*}$ suggests a mass $\approx \varepsilon e^{-t^{*}}=1 / \beta, e^{-\beta}$ even as $\varepsilon$ becomes small.

There are two basic Ward identities which hold for all $\beta, \varepsilon>0, q \in \mathbb{R}$,

$$
<e^{q t_{j}}>=<e^{(1-q) t_{j}}>\text { and } \sum_{x}<y_{0} y_{x}>=\varepsilon^{-1} .
$$

Note the similarity with the Ward identity (1.14) for the Green's function.

The following theorem gives a partial description of the ordered state of the $H^{2 \mid 2}$ model in 3D for large $\beta$. Let $G_{0}=(-\beta \Delta+\varepsilon)^{-1}$.

Theorem 3.1 ([DSZ]) In 3 or more dimensions for $\beta \geq \bar{\beta} \gg 1$ the fluctuations of the field $t$ are uniformly bounded in $x, y, \varepsilon$ and $\Lambda:\left\langle\cosh ^{m}\left(t_{x}-t_{y}\right)\right\rangle_{\Lambda, \beta, \varepsilon} \leq 2$ and the spin correlations satisfy

$$
\frac{1}{C}\left[f ; G_{0} f\right] \leq \sum_{j, k} f(j) f(k)\left\langle y_{j} y_{k}\right\rangle(\beta, \varepsilon) \leq C\left[f ; G_{0} f\right]
$$

for $f(j) \geq 0$.

Remarks: Note that (3.10) is similar to (2.22) for the hyperbolic sigma model. The lower bound in [SZ] and [DSZ] was improved by Y. Capdebosq, see [Sp3]. The 
proof Theorem (3.1) is different from that for the hyperbolic sigma model because the effective action is not convex.

The next theorem describes the disordered or localized phase of $H^{2 \mid 2}$ model for small $\beta$ in any dimension $d$.

Theorem $3.2([\mathrm{DS}])$ If $0<\beta<\bar{\beta}$ and $\bar{\beta}^{1 / 2} \log \left(\bar{\beta}^{-1}\right)<1 /(2 d-1)$, there is a finite localization length $\ell$ such that

$$
0 \leq<y_{0} y_{x}>(\beta, \varepsilon) \leq \frac{C_{\ell}}{\varepsilon} e^{-|x| / \ell(\beta)} .
$$

For a one dimensional chain $\ell(\beta) \leq C / \beta$ for all $\beta$.

Remarks: The estimate on $\bar{\beta}$ in the disordered phase in $3 \mathrm{D}$ is probably reasonably sharp. However, in the $3 \mathrm{D}$ ordered phase $\bar{\beta}$ is much larger than it is expected to be. Recent work of Disertori, Merkl and Rolles [DMR] establishes exponential localization for one dimensional strips of arbitrary width for any $\beta<\infty$. In [MR2] similar results were proved for the linearly edge reinforced random walk (ERRW) which is closely related to a variant of the $H^{2 \mid 2}$ model. In this case, the localization length is shown to be proportional to the width of the strip. In [MR1] a slow power law decay for the conductance was proved in 2D for the ERRW even for weak reinforcement. These results are similar to the power law obtained in [MS] for the 2D XY model. The proofs in [MR1, MR2, DMR, MS] use a deformation of the statistical mechanics measure and is related to Mermin-Wagner.

Hessian suggests localization in 2D? The Hessian of the action for $H^{2 \mid 2}$ model about $t_{j}=0$ is approximately given by the quadratic form in $\left\{v_{j}\right\}$ as follows:

$$
\beta \sum_{j}(\nabla v)_{j}^{2}-\sum_{j, k}(\nabla v)_{j}\left(\nabla G_{0}\right)^{2}(j-k)(\nabla v)_{k}+\frac{\varepsilon^{2}}{\beta^{2}} \sum_{j, k} v_{j} v_{k} G_{0}^{2}(j-k)
$$

where $G_{0}=(-\Delta+\varepsilon / \beta)^{-1}$. In $2 \mathrm{D}$ note that $\sum_{j}\left(\nabla G_{0}\right)^{2}(j)$ has a log divergence as $\varepsilon \rightarrow 0$. A similar expression holds about $\mathrm{t}=$ const. Since $\ln \operatorname{Det} D(t)$ is convex, the second term of (3.12) is negative for any t. The Fourier transform of (3.12) is $\left(\beta-g_{\varepsilon}(p)\right) p^{2}$. Thus the effective spin stiffness (coefficient of $p^{2}$ ) goes to 0 as $p$ and $\varepsilon \rightarrow 0$. In $3 \mathrm{D}, g_{\varepsilon}(p)$ is uniformly bounded thus there is only a minor correction to $\beta$. For the hyperbolic sigma model the first minus sign in (3.12) is plus so the effective spin stiffness grows in $2 \mathrm{D}$.

3.2. Ward identities and sketch of proof. The proof of Theorems 3.1 and 3.2 above relies on Ward identities which are a consequence of internal supersymmetry. We focus on the proof of Theorem 3.1 since it is technically more complicated. The main goal is to show that fluctuations of the form $\left\langle\cosh ^{m}\left(t_{j}-t_{k}\right)\right\rangle_{\Lambda, \beta, \varepsilon}$ are bounded using induction on $|j-k|$.

We state some Ward identities following [DSZ]. Let $\mathrm{S}$ be an integrable function of the variables $x, y, z, \xi, \eta$ which is supersymmetric, i.e., it is invariant under transformations preserving

$$
x_{i} x_{j}+y_{i} y_{j}+\xi_{i} \eta_{j}-\eta_{i} \xi_{j}
$$

then $\int S=S(0)$. In horospherical coordinates the function $S_{i j}$ given by

$$
S_{i j}=B_{i j}+e^{t_{i}+t_{j}}\left(\bar{\psi}_{i}-\bar{\psi}_{j}\right)\left(\psi_{i}-\psi_{j}\right), \quad B_{i j}=\cosh \left(t_{i}-t_{j}\right)+\frac{1}{2} e^{t_{i}+t_{j}}\left(s_{i}-s_{j}\right)^{2}
$$


is supersymmetric. If $\mathrm{i}$ and $\mathrm{j}$ are nearest neighbors, $S_{i j}-1$ is a term in the action $\mathcal{A}$ given in (3.4) and it follows that the partition function $Z_{\Lambda}(\beta, \varepsilon) \equiv 1$. More generally for each $m$ we have

$$
1=<S_{i j}^{m}>_{\beta, \varepsilon}=<B_{i j}^{m}\left[1-m B_{i j}^{-1} e^{t_{i}+t_{j}}\left(\bar{\psi}_{i}-\bar{\psi}_{j}\right)\left(\psi_{i}-\psi_{j}\right)\right]>_{\beta, \varepsilon} .
$$

Here we have used that $S_{i j}^{m} e^{-\mathcal{A}_{\beta, \varepsilon}}$ is integrable for $\varepsilon>0$. Since the action is quadratic in $\bar{\psi}, \psi$ the integration over the Grassmann variables is explicitly given using (2.4) by

$$
G_{i j}=\frac{\mathrm{e}^{t_{i}+t_{j}}}{B_{i j}}\left[\left(\delta_{i}-\delta_{j}\right) ; D_{\beta, \varepsilon}(t)^{-1}\left(\delta_{i}-\delta_{j}\right)\right]_{\Lambda} \quad \text { and } \quad 1 \leq \cosh ^{m}\left(t_{i}-t_{j}\right) \leq B_{i j}^{m} .
$$

Thus we have the identity

$$
<B_{i j}^{m}\left(1-m G_{i j}\right)>=1 .
$$

When $|i-j|=1$ it is easy to show that $0 \leq G_{i j}(t) \leq \beta^{-1}$ for all t. Then by (3.16)

$$
m \beta^{-1} \leq \frac{1}{2} \Rightarrow<\cosh ^{m}\left(t_{i}-t_{j}\right)>\leq<B_{i j}^{m}>\leq 2 .
$$

Remarks: The vector $\left(\delta_{i}-\delta_{j}\right)$ is orthogonal to the 0 mode of $D_{\beta, \varepsilon}(t)$ in finite volume containing $i$ and $j$. If we set $t_{k}=0, s_{k}=0$ for all $k$, then it is clear that $G_{i j}$ is uniformly bounded by $C \beta^{-1}$ for all $i, j \in \mathbb{Z}^{3}$. However, in two dimensions it diverges logarithmically in $|i-j|$. From the definition of $D_{\beta, \varepsilon}$ given in (3.5) we might expect that for large $\beta, \mathrm{G}$ in (3.15) is also of order $1 / \beta$ in $3 \mathrm{D}$. However, there are rare configurations of $t_{k} \ll-1$ with $k$ on a surface separating $\mathrm{i}$ and $\mathrm{j}$ for which $G_{i j}$ can diverge as $\varepsilon \rightarrow 0$. To see this consider $\mathrm{G}$ with Neumann boundary conditions separating i and $\mathrm{j}$. Then in a finite box $G_{j j}$ diverges as $\varepsilon$ goes to 0 .

For distances $|x-y|>1$, there is no uniform bound on $G_{x y}$. In 3D sufficient conditions on the field $t$ to get the estimate $G_{x y} \leq C / \beta$ are given by

$$
1 \leq \cosh \left(t_{j}-t_{x}\right) \leq B_{j x} \leq a|j-x|^{\alpha}, \quad 0<\alpha<1 / 2,
$$

and the same for $\cosh \left(t_{j}-t_{y}\right)$. The number $a$ is a constant, say $a>10$. It will turn out that these estimates are needed only for the sites $j$ in a 3D diamond-type region, $R_{x y}$, containing $x$ and $y$. Notice that since the exponent $\alpha$ is positive, we are allowing larger fluctuations at larger scales. The probability that such a condition is violated will be shown to be small by induction.

These conditions described above are initially expressed in terms of supersymmetric characteristic functions $\chi_{x, y}$ inserted in (3.16). It is important to show that the nilpotent (or Grassmann) part of $\chi_{x, y}$ is not important, so we may think of $\chi_{x, y}$ in the usual classical sense. The remaining problem is to obtain unconditional estimates on the fluctuations and thereby prove Theorem 3.1. This is first done for short scales. For larger scales we use induction.

Our induction hypothesis is that

$$
\left\langle\prod_{i=1}^{n} B_{x_{i} y_{i}}^{m}\right\rangle \leq 2^{n}
$$

holds under the assumption that the diamond-type regions associated with $i=$ $1, \ldots, n$ have disjoint interiors. The induction is in $\ell$, defined as the maximal separation $\left|x_{i}-y_{i}\right|$ in the product over $i=1, \ldots, n$. For $\ell=1$ this hypothesis is easily verified. 
In order to prove unconditional estimates on the fluctuations, first consider a site $b$ in $R_{x y}$ closest to $x$ or $y$ such that condition (3.18) is violated for $j=b$. We shall then prove by induction that the probability for such an event to occur is small. The inequality $B_{x y}^{m}<2^{m} B_{x c}^{m} B_{c y}^{m}$ is used for a point $c$ near $b$. Since the distances $|x-c|$ and $|c-y|$ are less than $|x-y|$, induction can be applied. The factor $2^{m}$ is offset by the small probability of the event when $\beta$ is large.

3.3. The Efetov sigma model. In this section we follow [Ef1, Fyo, Mir, Sp3, Zi1] to give a brief discussion of the Efetov sigma model. In order to obtain information about averages of the form $\left\langle\left|G\left(E_{\varepsilon} ; 0, j\right)\right|^{2}\right\rangle$ we introduce a field with four components

$$
\Phi_{j}=\left(z_{j}, w_{j}, \psi_{j}, \chi_{j}\right)
$$

where $z, w$ are complex fields and $\psi, \chi$ are Grassmann fields. Let $L=\operatorname{diag}(1,-1,1,1)$, and $\Lambda=\operatorname{diag}(1,-1,1,-1)$. For a Hermitian matrix $H$, define the action

$$
A(E, \varepsilon)=\Phi^{*} \cdot L\{i(H-E)+\varepsilon \Lambda\} \Phi .
$$

Note that the signature of $\mathrm{L}$ is chosen so that the $z$ and $w$ variables appear as complex conjugates of each other. Then we have the identity:

$$
\left|G\left(E_{\varepsilon} ; 0, j\right)\right|^{2}=\int z_{0} \bar{z}_{j} w_{0} \bar{w}_{j} e^{-A(E, \varepsilon)} D \Phi
$$

where

$$
D \Phi \equiv D z D w D \psi D \chi
$$

Without the observable $z_{0} \bar{z}_{j} w_{0} \bar{w}_{j}, \int e^{-A}=1$. The integral over Gaussian $\mathrm{H}$ can be calculated as in (2.6) and will produce a quartic interaction in $\Phi$. However, now the Hubbard-Stratonovich transformation, which is usually used in the Bosonic sector, involves a subtle analytic continuation first worked out in [SW], see also [Fyo, Zir1].

Now let us define a matrix of the form

$$
M=\left(\begin{array}{ll}
{[B B]} & {[B F]} \\
{[F B]} & {[F F]}
\end{array}\right)
$$

where each block is a $2 \times 2$ matrix. $\mathrm{M}$ will be called a supermatrix if the diagonal blocks, BB and FF are made of commuting (even elements) variables while the off diagonal blocks FB and BF consist of odd elements in the Grassmann algebra. Define the supertrace

$$
\operatorname{Str}(M) \equiv \operatorname{Tr}([B B]-[F F]) .
$$

Note that for supermatrices A and B we have $\operatorname{Str}(A B)=\operatorname{Str}(B A)$. We define the adjoint of a supermatrix $M$ by

$$
M^{\dagger}=\left(\begin{array}{cc}
{[B B]^{*}} & {[F B]^{*}} \\
-[B F]^{*} & {[F F]^{*}}
\end{array}\right) .
$$

The symbol * denotes the usual transpose followed by conjugation. For Grassmann variables we have $\overline{\psi_{a} \psi_{b}}=\bar{\psi}_{a} \bar{\psi}_{b}$. But $\bar{\psi}=-\psi$ so that $\dagger$ is an involution and

$$
\Phi_{1}^{\dagger}\left(M \Phi_{2}\right)=\left(M^{\dagger} \Phi_{1}\right)^{\dagger} \Phi_{2} .
$$

For $\varepsilon=0$ the action is invariant under the action of matrices $T$ in $S U(1 ; 1 \mid 2)$ which by definition satisfy:

$$
T^{\dagger} L T=L .
$$


As in $\S 2.5$ the sigma approximation of the spin is given by matrices

$$
S_{j}=T_{j}^{-1} \Lambda T_{j}
$$

as T ranges over $S U(1 ; 1 \mid 2)$. It is the orbit of a critical point, which is proportional to $\Lambda$ under the action of $S U(1,1 \mid 2)$. Thus the matrix $\mathrm{S}$ ranges over a supersymmetric space $U(1,1 \mid 2) /(U(1 \mid 1) \times U(1 \mid 1))$. The SUSY sigma model has a Gibbs density defined by

$$
\exp \left\{-\beta S \operatorname{tr} \sum_{j \sim j^{\prime}}\left(S_{j} S_{j^{\prime}}\right)-\varepsilon S \operatorname{st} \sum_{j} \Lambda S_{j}\right\} .
$$

If we drop the FB contribution we get the hyperbolic sigma model, (BB), and the ferromagnetic Heisenberg model, (FF). The sign of the action is correct in (3.24) but incorrect in [Sp3]. It is the FB contribution which couples these sectors and makes mathematical analysis difficult. A general discussion of supersymmetric spaces appears in [Zir3].

In a one dimensional chain of length $\mathrm{L}$ with $\varepsilon=0$ except at the end points, the Grassmann variables can be explicitly integrated over producing the formula (2.31). An explicit parametrization of the $4 \times 4$ matrices $S_{j}$ and integration measure is given in [Ef1, Mir]. As in $\S 2.5$ and the discussion of the Heisenberg model, fluctuations about the saddle should produce massless modes - Goldstone Bosons in 3D for the Efetov model which are responsible for quantum diffusion.

\section{Concluding Remarks}

(1) Universality of local eigenvalue spacing is well understood for many mean field models, [ER5]. In these lectures we have tried to present a picture for why one should expect universality of mean field theory in RBM and the Anderson model provided that the dual spin system is ordered. T. Shcherbina has recently applied SUSY statistical mechanics (with no approximations) to prove universality of $\rho_{2}$ for certain RBM made of GUE blocks [Shc3]. However, much more needs to be done to analyze the ordered phases of classical spin models with continuous symmetry and SUSY models whose 0 modes give Wigner-Dyson statistics.

(2) The average Green's function for a 3D Gaussian band matrix has been successfully analyzed in detail by the SUSY formalism. For weak disorder, smoothness of the density of states and exponential decay of $\left\langle G\left(E_{\varepsilon} ; j, k\right)\right\rangle$ have not been proved for the Anderson model in $3 \mathrm{D}$ due to strong oscillations produced by $-\Delta-E$ in the SUSY model.

(3) Although SUSY statistical mechanics provides a natural approach for the analysis of random band matrices and also for certain history dependent walks, results about eigenvectors and universality of eigenvalue spacing for random band matrices are still very limited. The most promising avenue of success is for one dimensional band matrices of width $\mathrm{W}$. When $W^{2} \gg N$ one expects that the eigenstates are extended, $[\mathrm{FM}]$, and that the eigenvalue spacing is given by GUE or GOE for $|E|<2-\delta, \delta>0$.

(4) In 2 dimensions we expect that all eigenstates are localized in the infinite volume limit. In statistical mechanics this means that even at low temperature the corresponding spin correlations decay exponentially fast at long distances. For example the Manhattan model, the vertex reinforced jump process (VRJP) and the Anderson model are expected to have localized dynamics for all non zero strengths 
of disorder. An exception to this localization conjecture are quantum systems with spin orbit coupling. Renormalization calculations predict these systems exhibit superdiffusion in 2D, [Ef1, Ef2].

(5) What happens at energies $E_{m}, E_{m}^{\prime}$ or temperature $T_{c}$ which mark the borderline between localization and diffusion? There has been much analysis in theoretical physics devoted to the multi-fractal behavior of eigenfunctions with energy near $E_{m}$, see for example [EM]. The behavior of localization near $E_{m}$ is poorly understood and there is no upper critical dimension. This is in contrast to the Ising or $\mathrm{XY}$ models which have Gaussian free field behavior at $T_{c}$ when the dimension $d \geq 4$. Fyodorov and Zirnbauer have suggested that the transition for the $H^{2 \mid 2}$ model may have many interesting features in three or more dimensions. This transition has been explored [DFZ], in the Migdal approximation.

\section{References}

[Abr] E. Abrahams, P. W. Anderson, D. C. Licciardello and T. V. Ramakrishnan, Scaling theory of localization: Absence of quantum diffusion in two dimensions, Phys. Rev. Lett. 42, 673677 (1979).

[AM] M. Aizenman and S. Molchanov, Localization at large disorder and at extreme energies: an elementary derivation, Commun. Math. Phys. 157, 245-278 (1993).

[And] P. W. Anderson, Absence of Diffusion in Certain Random Lattices, Phys. Rev. 109, 14921505 (1958).

[Apo] T. Apostol, Modular Functions and Dirichlet Series in Number Theory, 41, Graduate Texts in Mathematics, Springer Verlag.

[Bal] T. Balaban, A low temperature expansion for classical N-vector models. I. A renormalization group flow, Commun. Math. Phys. 167 103-154 (1995).

[Ber] F. A. Berezin, Introduction to Superanalysis, (Reidel, Dordrecht, The Netherlands, 1987).

[BEY] P. Bourgade, L. Erdős, H.-T. Yau, Universality of general $\beta$-Ensembles, arXiv:1104.2272.

[Bre] E. Brezin, V. Kazakov, D. Serban, P. Wiegman, A. Zabrodin, Applications of Random matrices to physics, Nato Science Series 221, Springer 2006.

[BL] H. Brascamp and E. Lieb, On extensions of the Brunn-Minkowski and Prekopa-Leindler theorems, including inequalities for log concave functions, and with an application to the diffusion equation, J. Func. Anal. 22, 366-389 (1976).

[BOC] E. J. Beamond, A. L. Owczarek, J. Cardy, Quantum and classical localisation and the Manhattan lattice, J. Phys. A, Math. Gen. 3610251 (2003), arXiv:cond-mat/0210359.

[Car] J. Cardy, Quantum Network Models and Classical Localization Problems, Int. J. Mod. Phys. B, 24, 1989-2014 (2010).

[Con] F. Constantinescu, G. Felder, K. Gawedzki, A. Kupiainen, Analyticity of density of states in a gauge-invariant model for disordered electronic systems, Jour. Stat. Phys. 48, 365-391 (1987).

[Dei] P. Deift, T. Kriecherbauer, K. T.-K. McLaughlin, S. Venakides, X. Zhou, Uniform asymptotics for polynomials orthogonal with respect to varying exponential weights and applications to universality questions in random matrix theory, Comm. Pure Appl. Math. 52, 1335-1425 (1999).

[Dis] M. Disertori, Density of states for GUE through supersymmetric approach, Rev. Math. Phys. 16, 1191-1225 (2004).

[DFZ] W. Drunk, D. Fuchs, M.R. Zirnbauer, Migdal-Kadanoff renormalization of a nonlinear supervector model with hyperbolic symmetry, Ann. Physik 1 134-150 (1992).

[DMR] M. Disertori, F. Merkl, S. Rolles, Localization for a nonlinear sigma model in a strip related to vertex reinforced jump processes, preprint.

[DPS] M. Disertori, H. Pinson, T. Spencer, Density of States for Random Band Matrices, Commun. Math. Phys. 232, 83-124 (2002), arxiv.org/math-ph/0111047.

[DS] M. Disertori, T. Spencer, Anderson Localization for a SUSY sigma model, Commun. Math. Phys. 300 659-671 (2010), arXiv.org/abs/0910.3325.

[DSZ] M. Disertori, T. Spencer, M. R. Zirnbauer, Quasi-diffusion in a 3D Supersymmetric Hyperbolic Sigma Model, Commun. Math Phys., 300, 435-486 (2010); arxiv.org/abs/0901.1652. 
[Dys] F.J. Dyson, Statistical Theory of the Energy Levels of Complex Systems. I, II, III, J. Math. Phys. 3, 140-175 (1962).

[Ef1] K.B. Efetov, Supersymmetry and theory of disordered metals, Adv. Phys.32, 874 (1983).

[Ef2] K.B. Efetov, Supersymmetry in Disorder and Chaos, (Cambridge, UK, Cambridge University Press, 1997).

[Ef3] K.B. Efetov, Minimum Metalic conductivity in the theory of localization, JETP Lett, 40, 738-741 (1984).

[Ef4] K.B. Efetov, Anderson Localization and Supersymmetry, In: 50 years of Anderson Localization, ed. by E. Abrahams, World Scientific Publishing Company 2010, arXiv.org/abs/1002.2632.

[Er1] L. Erdős, M Salmhofer, H.-T. Yau, Quantum diffusion of the random Schrödinger evolution in the scaling limit, Acta Mathematica 200, 211-277 (2008).

[Er2] L. Erdős S. Peche, J. Ramírez, B. Schlein, and H-T. Yau, Bulk universality for Wigner matrices. Commun. Pure Appl. Math. 63, No. 7, 895-925 (2010).

[Er3] L. Erdős, A. Knowles, H.-T. Yau, J. Yin Delocalization and Diffusion Profile for Random Band Matrices, preprint, arXiv:1205.5669v3.

[Er4] L. Erdős, H.-T. Yau, Universality of local spectral statistics of random matrices. Bull. Amer. Math. Soc. 49, 377-414 (2012).

[Er5] L. Erdsős, Universality for random matrices and log-gases, preprint, arXiv:1212.0839.

[EM] F. Evers, A.D. Mirlin, Anderson Transitions, Rev. Mod. Phys. 80, 1355-1417 (2008).

[FP] J. Fröhlich, C. Pfister, Spin Waves, Vortices, and the Structure of Equilibrium States in the Classical X Y Model, Commun. Math. Phys. 89, 303-327 (1983).

[FS1] J. Fröhlich, T. Spencer, The Kosterlitz-Thouless Transition, Commun. Math. Phys. 81, 527-602 (1981).

[FS2] J. Fröhlich and T. Spencer, Absence of diffusion in the Anderson tight binding model for large disorder or low energy, Comm. Math. Phys. 88 (1983), 151-184.

[FSS] J. Fröhlich, B. Simon, T. Spencer, Infrared bounds, phase transitions and continuous symmetry breaking, Commun. Math. Phys. 50 75-95 (1976).

[FM] Y.V. Fyodorov and A.D. Mirlin, Scaling properties of localization in random band matrices: a $\sigma$-model approach, Phys. Rev. Lett. 67, 2405-2409, (1991).

[Fyo] Y.V. Fyodorov, Basic Features of Efetov's SUSY. In: Mesoscopic Quantum Physics, (Les Houches, France, 1994), ed. by E. Akkermans et al.

[GLR ] I.A. Gruzberg, A.W.W. Ludwig, N. Read, Exact exponents for the spin Quantun Hall transition, Phys. Rev. Lett. 82, 4524-4527 (1999).

[GMP] I. Goldsheid, S. Molchanov and L. Pastur, One-dimensional random Schrödinger operator has a pure point spectrum, Funct. Anal. Appl. 11, 1-10 (1977).

[HR] G. H. Hardy and S. Ramanujan, Asymptotic Fomulae in Combinary Analysis, Proc. London Math. Soc. series 2, 17, 75-115 (1918).

[KS] J.P. Keating and N.C. Snaith, Random Matrix Theorey and L functions at $s=1 / 2$, Commun. Math. Phys. 214, 91-110 (2000).

[KM] V.E. Kravtsov and A.D. Mirlin, Level statistics in a metallic sample: Corrections to the Wigner-Dyson distribution JETP Lett., 60, 645-649 (1994).

$[\mathrm{KT}] \quad$ J.M. Kosterlitz and D.J. Thouless, Ordering, metastability and phase transitions in twodimensional systems, J. Phys. C 6, 1181 (1973).

[Mir] A.D. Mirlin, Statistics of energy levels. In: New Directions in Quantum Chaos, (Proceedings of the International School of Physics "Enrico Fermi", Course CXLIII), ed. by G.Casati et al. (IOS Press, Amsterdam), 223-298 (2000), arXiv.cond-mat/0006421.

[MR1] F. Merkl and S.W.W. Rolles, Bounding a Random Environment for Two-dimensional Edge-reinforced Random Walk, Elec. J. of Probability 13 530-565 (2008).

[MR2] F. Merkl and S.W.W. Rolles. Edge-reinforced random walk on one-dimensional periodic graphs. Probab. Theory Related Fields, 145(3-4):323-349 (2009).

[MS] O. McBryan, T. Spencer, On the decay of dorrelations in $\mathrm{SO}(\mathrm{n})$ symmetric ferromagnets, Commun. Math. Phys. 53, 299-302 (1977).

[OSC] M. Ortuño, A. M. Somoza and J. T. Chalker, Random Walks and Anderson Localization in a Three-Dimensional Class C Network Model, Phys. Rev. Lett. 102, 070603-7 (2009).

[PS1] L. Pastur, M. Shcherbina; Universality of the local eigenvalue statistics for a class of unitary invariant random matrix ensembles. J. Stat. Phys. 86, 109-147 (1997). 
[PS2] L. Pastur and M. Shcherbina; Eigenvalue distribution of large Random Matrices; Mathematical Surveys and Monographs 171, American Mathematical Society (2011).

[Pol] A.M. Polyakov, Interaction of Goldstone particles in two dimensions. Phys. Lett. 59B 79-81 (1975).

[Rad] H. Rademacher, On the Expansion of the Partition Function in a Series, Ann. of Math. 44 416-422 (1943).

[RS] Z. Rudnick and P. Sarnak, Zeros of principal L-functions and random-matrix theory, Duke Math. J. 81, 269-322 (1996).

[Sal] M. Salmhofer, Renormalization: An Introduction, (Springer, 1999).

[Sch] J. Schenker, Eigenvector Localization for Random Band Matrices with Power Law Band Width, Commun. Math. Phys. 290, 1065-1097 (2009).

[Sha] M. Shamis, Density of States for GUE and GOE through a supersymmetric approach, preprint.

[Shc1] T. Shcherbina, On the correlation function of the characteristic polynomials of the hermitian Wigner ensemble, Commun. Math. Phys., 308, 1-21 (2011); http://arxiv.org/abs/1006.2536v1.

[Shc2] T. Shcherbina On the second mixed moment of the characteristic polynomials of the 1D band matrices, http://arxiv.org/abs/1209.3385v3.

[Shc3] T. Shcherbina, Universality of the local regime for the block band matrices with a finite number of blocks, preprint.

[Sod] A. Sodin, The spectral edge of some random band matrices, Annals of Mathematics, 172, $2223(2010)$.

[Sp1] T. Spencer, Mathematical aspects of Anderson Localization. In: 50 years of Anderson Localization, ed. by E. Abrahams (World Scientific Publishing Company, 2010).

[Sp2] T. Spencer, Random Band and Sparse Matrices, ed. G. Akemann, J. Baik, P. Di Francesco, in The Oxford Handbook of Random Matrix Theory (Oxford University Press, 2010).

[Sp3] T. Spencer, SUSY Statistical Mechanics and Random Band Matrices, In Quantum Theory from Small to Large Scales edited by J. Fröhlich et al.; Oxford University Press, 2012.

[ST] C. Sabot and P. Tarres, Edge-reinforced random walk, Vertex-Reinforced Jump Process and the supersymmetric hyperbolic sigma model, http://arxiv.org/abs/1111.3991.

[Sto] G. Stolz, An Introduction to the Mathematics of Anderson Localization, Contemporary Mathematics 552, 71-108 AMS (2011), arXiv:1104.2317.

[SW] L. Schäfer and F. Wegner, Disordered system with $\mathrm{n}$ orbitals per site: Lagrange formulation, hyperbolic symmetry, and Goldstone modes Z. Phys. B 38, 113-126 (1980).

[SZ] T. Spencer and M. R. Zirnbauer, Spontaneous symmetry breaking of a hyperbolic sigma model in three dimensions, Comm. Math. Phys.252, 167-187 (2004).

[TV] Tao, T. and Vu, V.: Random matrices: Universality of the local eigenvalue statistics. Acta Math., 206, 127-204 (2011).

[VWZ] J.J.M. Verbaarschot, H.A. Weidenmüller, and M.R. Zirnbauer. Phys. Reports, 129, 367438 (1985).

[Weg] F. Wegner, The Mobility edge problem: Continuous symmetry and a Conjecture, Z. Phys. B 35, 207-210 (1979).

[Wig1] E. P. Wigner, Characteristic vectors of bordered matrices with infinite dimensions. Ann. of Math. 62, 548-564 (1955).

[Wig2] E. P. Wigner, Random Matrices in Physics Siam Review 9 1-23, (1967).

[Zir1] M. R. Zirnbauer, The Supersymmetry method of random matrix theory, http://arXiv.org/abs/math-ph/0404057 (2004).

[Zir2] M. R. Zirnbauer, Fourier analysis on a hyperbolic supermanifold with constant curvature, Commun. Math. Phys. 141, 503-522 (1991).

[Zir3] M. R. Zirnbauer, Riemannian symmetric superspaces and their origin in random-matrix theory, J. Math. Phys. 37, 4986-5018 (1996).

Institute for Advanced Study, Princeton, New Jersey, U.S.A.

E-mail address: spencer@math.ias.edu 